\title{
2 Symmetry Based Fundamentals of Carbon Nanotubes
}

\author{
M. Damnjanović, I. Milošević, E. Dobardžić, T. Vuković, and B. Nikolić
}

Due to pioneering work of Eugen Wigner, Symmetry is well recognized as a clue to understanding physical processes. However, except in Particle Physics, role of Symmetry is mostly conceptual, to give a posteriori profound interpretation of the obtained results. For instance, in Solid State Physics, more than a century known crystal symmetries are applied to describe phase transitions, selection rules, tensor shapes. Probably the only exception is the Bloch theorem, which gives a priori general forms of the electronic quantum states and ionic displacements. However, this theorem treats only the translational symmetry, reducing the calculations to the elementary cell, which is, in many cases, far bellow the maximal possible reduction.

Over the past decade nanotubes have become one of the most attractive subjects in Condensed Matter Physics. Elementary cell of nanotubes often contains large number of atoms, which makes the Bloch theorem based calculations very robust, and frequently too demanding. Nevertheless, besides the translational symmetry, quasi-1D-crystals have helical and additional symmetries (like horizontal two-fold axes and/or mirror planes), enabling to generate the whole compound from the so called symmetry cell (symcell), being only a small part of the conventional unit cell. It is intuitively obvious that the physical properties of the system are determined by its symmetry group and by the symcell. Modified group projector technique offers an algorithm to maximally generalize the Bloch theorem to the full symmetry group. It also emphasizes that symmetry itself is the most essential property of nanotubes which proves to be sufficient to predict their unique physical properties.

\subsection{Introduction}

In this chapter, within a profound and efficient symmetry based approach, an overview of the electro-optical and vibrational properties of carbon nanotubes (NT) is presented.

In Sect. 2.2 the basics of single-wall tubes (SWNT) are introduced: configuration, chirality, symmetry and conserved quantum numbers. The stable configurations of narrow tubes are obtained by the symmetry preserving density functional relaxation. Afterwards the configuration of double-wall tubes is described. 
Section 2.3 starts with an outline of the concept necessary to exploit full symmetry in the band structure calculations and proceeds by giving a unified description of electron and phonon dispersions: band degeneracy, symmetry assignation, systematic density of state singularities and selection rules for various processes. The corresponding generalized Bloch eigenfunctions are also listed. Finally, dynamical models are reviewed and discussed: $p$ - and $s p^{3}$ orbitals are tight-binding and density functionals for electrons, while phonons are studied by intra-layer force constants and (for double-wall tubes) interlayer Lenard-Jones interactions.

Electro-optical properties of SWNTs are studied in Sect. 2.4. Polarized optical absorption of conventional (with the diameters $8-16 \AA$ ) and template grown ( $4 \AA$-diameter) SWNTs are calculated within first order timedependent perturbation theory, using exactly evaluated optical transition matrix elements out of the full-symmetry adapted Bloch eigenfunctions. The results obtained are discussed and compared to the measured spectra.

Vibrational properties of NTs are discussed in Sect. 2.5. After description of the phonon dispersions of SWNTs (emphasizing some particularly interesting modes), double-wall tube branches are considered. The so called rigid layer modes are interpreted with the help of the simple perturbative model. Also, some related properties, such as heat capacity, sound velocities, overbending, Raman and infrared activity are discussed.

Finally, Sect. 2.6 is devoted to the interaction between the walls in doublewall NTs, which is calculated by the use of the expansion over symmetry based harmonics. Besides the high efficiency, the method profoundly correlates interaction with the breaking of the symmetry of the layers, explaining experimentally verified weak inter-layer interaction, and yielding, in some ideal cases, super slippery sliding.

\subsection{Configuration and Symmetry}

Symmetry of SWNTs is considered from their very discovery [1] due to its relevance for understanding physical properties of SWNTs. At first, only the rotational axis of the related fullerene molecule is observed [3]; later, in the electronic band calculations the translational [4], as well as the helical [5,6] symmetry was used. Finally, the full symmetry groups, including parities in addition to the roto-helical symmetries, were found [7]. These considerations are based on infinite, defect-free model of SWNTs corresponding to simply rolled up graphene layer.

\subsubsection{Single-Wall Nanotubes}

\section{Symmetry}

The graphene layer is a highly symmetric structure, with translational periodicity in two directions, a six-fold principle rotational axis and six mirror 


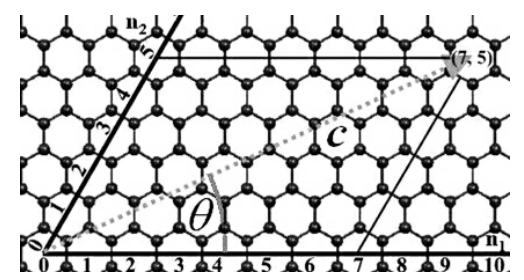

Fig. 2.1. Graphene honeycomb with depicted chiral vector $\boldsymbol{c}$ and chiral angle $\theta$

planes perpendicular to the layer (the horizontal plane and $U$-axes are not relevant in this context). To obtain the SWNT $\left(n_{1}, n_{2}\right)$, one takes the chiral vector $\boldsymbol{c}=\left(n_{1}, n_{2}\right)$ on the graphene (Fig. 2.1), and rolls up the layer so that this vector becomes the circumference of the cylinder. The special cases $(n, 0)$ and $(n, n)$ are known as the achiral zig-zag $(\mathcal{Z})$ and $\operatorname{armchair}(\mathcal{A})$ tubes, while the other ones are called chiral $(\mathcal{C})$ tubes. The folding changes the symmetry of the structure: the translations perpendicular to the chiral vector remain translations of the tube, those along $\boldsymbol{c}$ become rotational symmetries of the tube, while the combined ones give nanotube helical symmetries. After folding, the graphene principle axis is no longer symmetry, except that the rotations for $\pi$ become the $U$ axis of the tube. Finally, only in the achiral cases, two perpendicular mirror planes are preserved, becoming vertical $\left(\sigma_{\mathrm{v}}\right)$ and horizontal $\left(\sigma_{\mathrm{h}}\right)$ mirror planes of the zig-zag and armchair tubes. Combining all the symmetry transformations of the obtained tube results in its full symmetry group.

Since the translations perpendicular to the chiral vector $\left(n_{1}, n_{2}\right)$ on graphene remain the translational symmetries of the tube $\left(n_{1}, n_{2}\right)$, SWNTs are quasi one-dimensional crystals, and therefore their symmetries are gathered in the line groups describing the symmetries of such crystals $[8,9]$. A straightforward calculation [7] shows that the obtained line group is:

$$
\boldsymbol{L}_{\mathcal{C}}=\boldsymbol{T}_{q}^{r}(a) \boldsymbol{D}_{n}=\boldsymbol{L} q_{p} 22, \quad \boldsymbol{L}_{\mathcal{Z A}}=\boldsymbol{T}_{2 n}^{1}(a) \boldsymbol{D}_{n h}=\boldsymbol{L} 2 n_{n} / m c m
$$

where the parameters $n$ (order of the principle axis), $a$ (translational period), $q$ (one half of the number of atoms per period) and $r$ (helicity) are:

$$
\begin{array}{ll}
n=\operatorname{GCD}\left(n_{1}, n_{2}\right), & a=\frac{\sqrt{3\left(n_{1}^{2}+n_{2}^{2}+n_{1} n_{2}\right)}}{n \mathcal{R}} a_{0} \\
q=2 \frac{n_{1}^{2}+n_{1} n_{2}+n_{2}^{2}}{n \mathcal{R}}, & r=\frac{n_{1}+2 n_{2}-\left(\frac{n_{2}}{n}\right)^{\phi\left(\frac{n_{1}}{n}\right)-1} q \mathcal{R}}{n_{1} \mathcal{R}} \quad\left(\bmod \frac{q}{n}\right)(2.3
\end{array}
$$

$\left(\mathcal{R}=\operatorname{GCD}\left(2 n_{1}+n_{2}, n_{1}+2 n_{2}\right) / n, a_{0}=2.46 \AA\right.$ is graphene period, $\phi(x)$ is the Euler function, i.e. the number of coprimes not greater than $x$ ). In international notation helicity is characterized by

$$
p=n\left(r^{\phi\left(\frac{q}{n}\right)-1} \quad\left(\bmod \frac{q}{n}\right)\right) .
$$




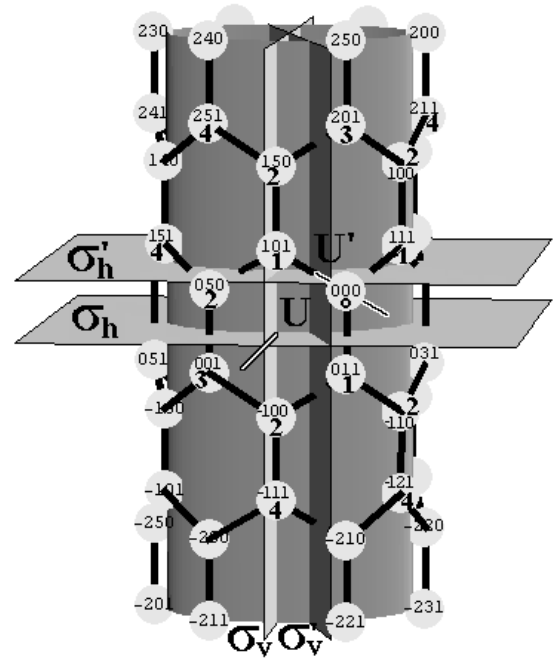

Fig. 2.2. Nanotube $(6,0)$. Symmetries: vertical and horizontal mirror planes $\sigma_{\mathrm{v}}$ and $\sigma_{\mathrm{h}}$ intersect along the horizontal rotational axis $U$; glide plane $\sigma_{\mathrm{v}}^{\prime}$ and rotoreflectional plane $\sigma_{\mathrm{h}}^{\prime}$ intersect along $U^{\prime}$. Atoms: labelled by tsu indices from (2.9). Neighbors of $\mathrm{C}_{000}$ : first to fourth level denoted by 1-4. Coordinate frame: $x$-axis coincides with $U$ ( $z$-axis is the tube axis)

Note that for achiral tubes $q=2 n, r=1$, and $a_{\mathcal{Z}}=\sqrt{3} a_{0}, a_{\mathcal{A}}=a_{0}$. The helical group $\boldsymbol{T}_{q}^{r}(a)$ is generated by $\left(C_{q}^{r} \mid \frac{n}{q} a\right)$, i.e. the rotation for $2 \pi r / q$ around the tube axis (z-axis) followed by the translation for $n a / q$ along it. The generators of the point group $\boldsymbol{D}_{n}$ are the rotation $\boldsymbol{C}_{n}$ for $2 \pi / n$ around the tube, and the rotation $U$ for $\pi$ around the $x$-axis (through the center of the carbon hexagon, Fig. 2.2). The achiral tubes point group $\boldsymbol{D}_{n h}$ has the additional generator $\sigma_{\mathrm{v}}$. Therefore, the symmetry transformations of SWNT are:

$$
\ell_{t s u}=\ell_{t s u}^{\mathcal{C}}=\left(C_{q}^{r} \mid \frac{n}{q} a\right)^{t} C_{n}^{s} U^{u}, \quad \ell_{t s u v}^{\mathcal{Z} \mathcal{A}}=\ell_{t s u} \sigma_{\mathrm{v}}^{v},
$$

with $t=0, \pm 1, \ldots, s=0, \ldots, n-1$ and $u, v=0,1$. All rotational, translational and helical symmetries (appearing due to the graphene translations) form the roto-translational subgroup $\boldsymbol{L}^{\mathrm{R}}=\boldsymbol{T}_{q}^{r}(a) \boldsymbol{C}_{n}$ including the elements $\ell_{t s 0}$. Among other transformations there are also radial $U$ axes bisecting the $\mathrm{C}-\mathrm{C}$ bonds, as well as various glide and roto-reflection planes (of achiral tubes). Applying only elements $\ell_{0 s u}$ onto the initial atom, one gets a monomer, i.e. the part of SWNT of the length $n a / q$, from which the helical transformations alone generate the whole tube.

SWNT symmetry groups are non-symmorphic, and their isogonal point groups, being important for the description of various physical tensors, are:

$$
\boldsymbol{P}_{\mathcal{C}}^{\mathrm{I}}=\boldsymbol{D}_{q}, \quad \boldsymbol{P}_{\mathcal{Z} \mathcal{A}}^{\mathrm{I}}=\boldsymbol{D}_{2 n h}
$$




\section{Configuration}

The number of symmetries in the chiral nanotubes is equal to the number of atoms, while in the achiral cases it is twice as big. This is extremely large symmetry. In fact, SWNTs are maximally symmetric structures in the sense that they are generated by symmetry operations from an arbitrary initial atom. This property can be used to parameterize the configuration in terms of symmetry. At first, the diameter and the linear density are:

$$
D=\frac{a n \mathcal{R}}{\pi \sqrt{3}}, \quad \lambda=\frac{2 q}{a}=c D
$$

$(c=14.375 \mathrm{amu} / \AA)$. Taking for the initial atom $\mathrm{C}_{000}$ with the cylindrical coordinates (Fig. 2.2)

$$
\boldsymbol{r}_{000}=\left(\frac{D}{2}, \varphi_{000}=2 \pi \frac{n_{1}+n_{2}}{n q \mathcal{R}}, z_{000}=\frac{n_{1}-n_{2}}{\sqrt{6 n q \mathcal{R}}} a_{0}\right),
$$

any other atom $\mathrm{C}_{t s u}$ is obtained by the action of $\ell_{t s u}$ on $\mathrm{C}_{000}$ :

$$
\boldsymbol{r}_{t s u}=\ell_{t s u} \boldsymbol{r}_{000}=\left(\frac{D}{2},(-1)^{u} \varphi_{000}+2 \pi\left(\frac{r t}{q}+\frac{s}{n}\right),(-1)^{u} z_{000}+t \frac{n}{q} a\right) .
$$

The described graphene folding structural model tacitly assumes that the SWNTs chemical bonds are based on the graphene $s p^{2}$ hybridization. However, due to curvature, $s p^{3}$ hybridization occurs (see Sect. 2.3.4). Therefore, the rolled up configuration is not stable, and should be relaxed (slightly changed) to achieve the local minimum of the total energy. Relaxation cannot change the symmetry: according to the famous topological theorem [10], the extremes of the energy functional correspond to the most symmetric configurations. Consequently, equations (2.1) and (2.9) refer to the relaxed structures as well, only the values of $D, \varphi_{000}, z_{000}$ and $a$ differ. Any change of these parameters preserves the symmetry of chiral tubes, except that $\varphi_{000}=\pi / n$ for zig-zag and $z_{000}=0$ for armchair tubes must be fixed. Obviously, the curvature induced rehybridization is significant only for narrow tubes. This is confirmed by precise density functional (DF) methods: only for tubes with a diameter of less than $7 \AA$ does the relaxed configuration differ from the rolled up one by a few percent (see Table 2.1), while the properties of thicker tubes are well described within the purely rolled up model.

\subsubsection{Double-Wall Nanotubes}

Double-wall tube $\mathrm{W} @ \mathrm{~W}^{\prime}$ is assumed to be a pair of coaxially arranged singlewall tubes: $\mathrm{W}=\left(n_{1}, n_{2}\right)$ is the inner wall and $\mathrm{W}^{\prime}=\left(n_{1}^{\prime}, n_{2}^{\prime}\right)$ is the outer wall. The inter-wall separation $\Delta=\left(D^{\prime}-D\right) / 2$ is close to the graphite interlayer distance $3.44 \AA$ within some tolerance $\delta$ (we use $\delta=0.25 \AA$ ). Fixing 

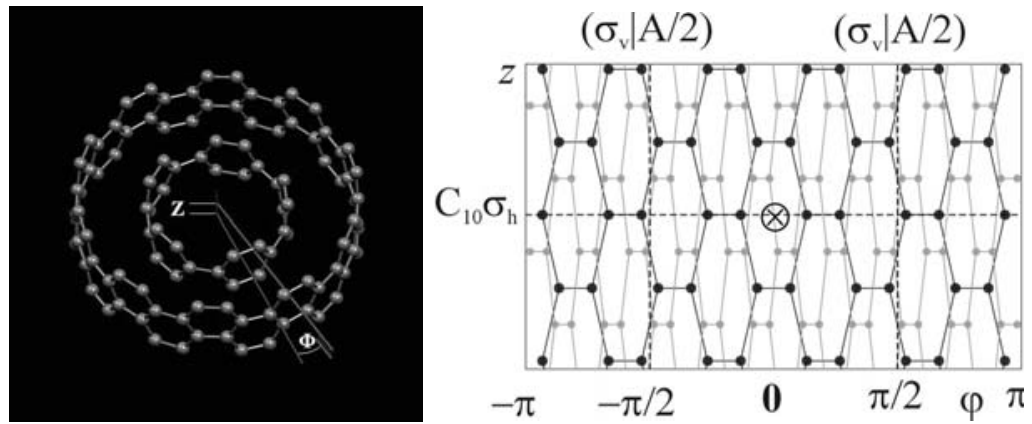

Fig. 2.3. Left: DWNT configuration. Right: unfolded tube $(5,5) @(10,10)$ with symmetry elements

Table 2.1. Ultra narrow tubes considered in Sect. 2.4.2. Folded configuration parameters (raw F) given by (2.8) and (2.2) are compared to the relaxed ones obtained by density functional tight-binding (raw DFTB, used in this chapter) and local density (raw DFLD taken from [11]) methods.

\begin{tabular}{|c|c|c|c|c|}
\hline SWNT & $D[\stackrel{\mathrm{A}}{0}]$ & $a[\stackrel{\mathrm{A}}{a}]$ & $\varphi_{000}\left[^{\circ}\right]$ & $z_{000}[\stackrel{\mathrm{A}}{\mathrm{A}}]$ \\
\hline$(5,0) \mathrm{F}$ & 3.92 & 4.26 & 36 & 0.71 \\
\hline DFTB & 4.11 & 4.23 & 36 & 0.70 \\
\hline DFLD & 4.12 & 4.30 & 36 & 0.73 \\
\hline$(3,3) \mathrm{F}$ & 4.07 & 2.46 & 40 & 0 \\
\hline DFTB & 4.19 & 2.48 & 40 & 0 \\
\hline DFLD & 4.24 & 2.50 & 40 & 0 \\
\hline$(4,2) \mathrm{F}$ & 4.15 & 11.28 & 39 & 0.27 \\
\hline DFTB & 4.29 & 11.27 & 39 & 0.26 \\
\hline DFLD & 4.34 & 11.34 & 39 & 0.28 \\
\hline$(5,1) \mathrm{F}$ & 4.36 & 23.73 & 35 & 0.51 \\
\hline DFTB & 4.51 & 23.67 & 35 & 0.51 \\
\hline DFLD & 4.56 & 24.18 & 35 & 0.52 \\
\hline
\end{tabular}

the interior wall, the configuration is completely determined by the twisting angle $\Phi$ and the axial shift $Z$ of the outer wall: the $x$-axis of the interior wall (defined in Fig. 2.2) should be rotated for $\Phi$ and shifted upward by $Z$ to get the $x$-axis of the outer wall (Fig. 2.3). Therefore, the atomic coordinates are (2.9) for $\mathrm{W}$, while for $\mathrm{W}^{\prime}$ :

$$
\boldsymbol{r}_{t^{\prime} s^{\prime} u^{\prime}}=\left(\frac{D^{\prime}}{2},(-1)^{u^{\prime}} \varphi_{000}^{\prime}+2 \pi\left(\frac{r^{\prime} t^{\prime}}{q^{\prime}}+\frac{s^{\prime}}{n^{\prime}}\right)+\Phi,(-1)^{u^{\prime}} z_{000}^{\prime}+t^{\prime} \frac{n^{\prime}}{q^{\prime}} a^{\prime}+Z\right) .
$$

Here, $q^{\prime}, r^{\prime}, n^{\prime}$ and $a^{\prime}$ are the parameters (2.2) of the symmetry group (2.1) of the outer wall.

The stable configuration of $\mathrm{W} @ \mathrm{~W}^{\prime}$ in such a model corresponds to the values of $\Phi$ and $Z$ minimizing the inter-layer potential: 


$$
V(\Phi, Z)=\sum_{t^{\prime} s^{\prime} u^{\prime}} \sum_{t s u} v\left(\boldsymbol{r}_{t s u}, \boldsymbol{r}_{t^{\prime} s^{\prime} u^{\prime}}^{\prime}\right)
$$

where $v\left(\boldsymbol{r}_{t s u}, \boldsymbol{r}_{t^{\prime} s^{\prime} u^{\prime}}^{\prime}\right)$ is the pairwise interaction of the interior- and outer-wall carbon atoms. Irrespectively of $v$, according to the topological argument [10], the optimal relative position is among the maximally symmetric ones. In fact, the symmetry group of $\mathrm{W} @ \mathrm{~W}^{\prime}$ contains only those transformations which simultaneously leave both walls invariant. Note that the common rotations, translations and helical symmetries are not affected by the relative positions of the walls, to differ from $U, \sigma_{\mathrm{v}}, \sigma_{\mathrm{h}}$, glide and roto-reflection symmetries appearing only when the corresponding axes and planes of the two walls coincide. Consequently, the candidates for the stable position are only those pairs $(\Phi, Z)$ enabling as much as possible coinciding parities. This singles out rather few a priori known possible stable configurations, reducing greatly the sensitivity of the choice of the potential model. This emphasizes the importance of symmetry of DWNTs.

At first, we consider $\Phi$ and $Z$ independent roto-translational symmetry, described by the intersection of the walls' roto-translational subgroups $[7,12$, $13]$ :

$$
\boldsymbol{L}_{\mathrm{WW}}^{\mathrm{R}}=\boldsymbol{L}_{\mathrm{W}}^{\mathrm{R}} \cap \boldsymbol{L}_{\mathrm{W}^{\prime}}^{\mathrm{R}}=\boldsymbol{T}_{Q}^{R}(A) \boldsymbol{C}_{N} .
$$

The parameters of this group are [13]:

$$
N=\operatorname{GCD}\left(n, n^{\prime}\right), A=\hat{a}^{\prime} a=\hat{a} a^{\prime}, Q=\frac{N \sqrt{\tilde{q} \tilde{q}^{\prime}}}{\tau}, R=\frac{(r \hat{a} \tau+s \tilde{q}) Q}{q},
$$

where $\tilde{q}=q / n, \tilde{q}^{\prime}=q^{\prime} / n^{\prime}, \hat{a}=\sqrt{\tilde{q} / \operatorname{GCD}\left(\tilde{q}, \tilde{q}^{\prime}\right)}, \hat{a}^{\prime}=\sqrt{\tilde{q}^{\prime} / \operatorname{GCD}\left(\tilde{q}, \tilde{q}^{\prime}\right)}$, while $\tau=\sqrt{\tilde{q} \tilde{q}^{\prime}} / \operatorname{GCD}\left(\frac{r^{\prime} \hat{a} n-r \hat{a}^{\prime} n^{\prime}}{N}, \sqrt{\tilde{q} \tilde{q}^{\prime}}\right)$. Further, the equation for the helicity $R$ involves $s=\tau\left(r^{\prime} \hat{a}^{\prime} q-r \hat{a} q^{\prime}\right)\left(\left(n^{\prime} / N\right)^{\phi(n / N)}-1\right) / n \tilde{q} \tilde{q}^{\prime}$.

Particularly, if the periods of the two walls are incommensurate (i.e. $a / a^{\prime}$ is irrational), W@ $\mathrm{W}^{\prime}$ possesses no translational periodicity $(A=\infty)$, reducing the symmetry to the point group $\boldsymbol{L}_{\mathrm{WW}}^{\mathrm{R}}=\boldsymbol{C}_{N}$. In fact, the commensurate tubes (CDWNTs) are very rare, about $0.5 \%$ of all DWNTs allowed by the inter-layer distance. Precisely, with 1280 SWNTs having diameters 2.8-50 $\AA$ altogether 42236 pairs satisfy requirements for $\Delta$ and $\delta$; among all these DWNTs only 240 are commensurate. Note here that if both walls are chiral, the tubes $\left(n_{1}, n_{2}\right) @\left(n_{1}^{\prime}, n_{2}^{\prime}\right)$ and $\left(n_{1}, n_{2}\right) @\left(n_{2}^{\prime}, n_{1}^{\prime}\right)$ must be independently considered, since the spatial inversion does not relate them (as in the single-wall case, which enables us to consider only $n_{1}^{\prime} \geq n_{2}^{\prime}$ SWNTs). This increases the number of different CDWNTs found to 318. Among them, with achiral walls are zig-zag and armchair series $\mathcal{Z Z}_{n}=(n, 0) @(n+9)$ and $\mathcal{A} \mathcal{A}_{n}=(n, n) @(n+5, n+5)$.

Finally, to find numerically the stable configurations and full symmetry groups, the choice of the potential must be made. It is well known that the pairwise interaction $v$ is of the Van der Waals type, and several concrete forms 
are proposed in the literature $[14,15]$. Here we use the simplest Lenard-Jones one [14]:

$$
v(\boldsymbol{r})=-\frac{18.5426}{|\boldsymbol{r}|^{6}}+\frac{29000.4}{|\boldsymbol{r}|^{12}}
$$

The results are in accordance with the topological prediction (some details of calculations are clarified in Sect. 2.6): in all the cases there are coinciding $U$ axes of the walls, and when both walls are achiral there is also a symmetry plane (mirror, glide or roto-reflectional) of one wall coinciding with a symmetry plane of another wall. Particularly, for incommensurate DWNTs $\Phi=0$, while $Z$ is arbitrary (thus the super-slippery sliding of the walls is enabled, as discussed in Sect. 2.6). For CDWNTs with at least one chiral wall $\Phi=Z=0$; for $\mathcal{Z} \mathcal{Z}$ and $\mathcal{A} \mathcal{A}$ tubes $\Phi=0$ and $Z=A / 4$, except that $\Phi=\pi / 4 N$ and $Z=A / 4$ for $\mathcal{Z Z}_{9}$ and $\mathcal{A A}_{5}$. Accordingly, the symmetry groups of commensurate tubes are line groups:

$$
\boldsymbol{L}_{\mathrm{WW}}^{\mathcal{C}}=\boldsymbol{T}_{Q}^{R}(A) \boldsymbol{D}_{N}, \quad \boldsymbol{L}_{\mathrm{WW}}^{\mathcal{Z} \mathcal{Z}_{9}, \mathcal{A} \mathcal{A}_{5}}=\boldsymbol{T}_{c}(A) \boldsymbol{S}_{2 N}, \quad \boldsymbol{L}_{\mathrm{WW}^{\prime}}^{\mathcal{Z Z}, \mathcal{A} \mathcal{A}}=\boldsymbol{T}(A) \boldsymbol{D}_{N d} .
$$

The specific results for more than 200 CDWNTs, given in Table 2.2, show that DWNTs have drastically reduced roto-translational symmetry in comparison to their walls.

Table 2.2. CDWNTs with collinear chiral vectors. The outer diameter of the series shown in column 1 is within 9.6-50 $\mathrm{A}$ ) only for $n$ given in column 4 (without braced values). Columns 2 and 3 : line and isogonal groups; translational periods are in the

\begin{tabular}{|c|c|c|c|}
\hline CDWNTs & Line Group & Isogonal & $n$ \\
\hline \multirow[t]{4}{*}{$(n, 0) @(n+9,0)$} & $\boldsymbol{T}(\sqrt{3}) \boldsymbol{D}_{1 d}$ & $\boldsymbol{D}_{1 d}$ & $\begin{array}{l}4,5, \ldots, 62 \\
(6,9,12, \ldots, 60)\end{array}$ \\
\hline & $\boldsymbol{T}(\sqrt{3}) \boldsymbol{D}_{3 d}$ & $D_{3 d}$ & $\begin{array}{c}6,9,12, \ldots, \ldots 60 \\
(9,18, \ldots 54)\end{array}$ \\
\hline & $\boldsymbol{T}(\sqrt{3}) \boldsymbol{D}_{9 d}$ & $\boldsymbol{D}_{9 d}$ & $18,27, \ldots, 63$ \\
\hline & $\boldsymbol{T}_{c}(\sqrt{3}) \boldsymbol{S}_{18}$ & & 9 \\
\hline \multirow[t]{3}{*}{$(n, n) @(n+5, n+5)$} & $\boldsymbol{T}(1) \boldsymbol{D}_{1 d}$ & $\boldsymbol{D}_{1 d}$ & $\begin{array}{l}2,3, \ldots, 36(5,10 \\
15,20,25,30,35)\end{array}$ \\
\hline & $\boldsymbol{T}(1) \boldsymbol{D}_{5 d}$ & $\boldsymbol{D}_{5 d}$ & $10,15, \ldots, 35$ \\
\hline & $\boldsymbol{T}_{c}(1) \boldsymbol{S}_{10}$ & & 5 \\
\hline \multirow[t]{2}{*}{$\begin{array}{c}(3 n, 2 n) @(3 n+6,2 n+4) \\
(3 n+6,2 n+4) @(2 n+4,3 n+6)\end{array}$} & $\boldsymbol{T}_{2}^{1}(\sqrt{57}) \boldsymbol{D}_{1}$ & $D_{2}$ & $1,3, \ldots, 13$ \\
\hline & $\boldsymbol{T}(\sqrt{57}) \boldsymbol{D}_{2}$ & & $2,4, \ldots, 14$ \\
\hline \multirow{4}{*}{$\begin{array}{l}(4 n, n) @(4 n+8, n+2) \\
(4 n, n) @(n+2,4 n+8)\end{array}$} & $\boldsymbol{T}_{2}^{1}(\sqrt{7}) \boldsymbol{D}_{1}$ & $D_{2}$ & $\begin{array}{l}1,3, \ldots, 13 \\
1,3, \ldots, 11\end{array}$ \\
\hline & $\boldsymbol{T}(\sqrt{7}) \boldsymbol{D}_{2}$ & & $\begin{array}{c}2,4, \ldots, 12 \\
2,4, \ldots, 12(6)\end{array}$ \\
\hline & $\boldsymbol{T}_{14}^{3}(\sqrt{7}) \boldsymbol{D}_{1}$ & $D_{14}$ & 13 \\
\hline & $\boldsymbol{T}_{14}^{13}(\sqrt{7}) \boldsymbol{D}_{2}$ & & 6 \\
\hline $\begin{array}{l}(7 n, 3 n) @(7 n+7,3 n+3) \\
(7 n, 3 n) @(3 n+3,7 n+7) \\
\end{array}$ & $\boldsymbol{T}(\sqrt{237}) \boldsymbol{D}_{1}$ & $D_{1}$ & $1,2, \ldots, 7$ \\
\hline $\begin{array}{l}(8 n, n) @(8 n+8, n+1) \\
(8 n, n) @(n+1,8 n+8)\end{array}$ & $\boldsymbol{T}(\sqrt{219}) \boldsymbol{D}_{1}$ & $D_{1}$ & $1,2, \ldots, 7$ \\
\hline
\end{tabular}
units of $a_{0}=2.46 \AA$. 


\subsection{Symmetry Based Band Calculations}

Due to Wigner's pioneering work, the conceptual importance of symmetry in physics is well recognized. However, except in particle physics, it is commonly used a posteriori, to give a profound explanation of the results obtained. Particularly in solid state physics, crystal symmetries which have been known for more than a century, are applied in this way: description of phase transitions, selection rules for various processes, tensor shapes. Perhaps the only exception is Bloch's theorem, a priori giving the form of the electronic quantum states and ionic displacements; even this theorem treats the translational periodicity only, which significantly restricts possible symmetry based conclusions, although it reduces the calculations to the elementary cell. The elementary cells of SWNTs contain many $(2 q)$ atoms, which makes the Bloch theorem based calculations very robust, and frequently too demanding even for the best computers. Nevertheless, it is shown that nontrivial helical, rotational and $U$-axis symmetries suffice to generate a tube from a single atom. Here we briefly sketch the modified group projector technique, giving an algorithm to reduce the calculations to the full symmetry cell, or symcell, from which the structure is generated by a complete symmetry group. Besides the generalization of the Bloch theorem, it provides a priori assignation of the (electron/phonon) bands by all the conserved quantum numbers, enabling application of all possible selection rules for physical processes.

\subsubsection{Modified Wigner Projectors}

Within the quantum mechanical formalism, the dynamics of the system is determined by its Hamiltonian $H$, being an operator in the state space $\mathcal{H}$. Its eigenstates $|n\rangle$ and eigenvalues $E_{n}$ solve the time independent Schrodinger equation $H|n\rangle=E_{n}|n\rangle$. Besides a few analytically solvable cases, this eigenproblem is hard even for a numerical approach, especially for complex systems. Below we describe the symmetry to simplify calculations.

Symmetries of a system, comprised in the group $\boldsymbol{L}$, are in the state space represented by the unitary operators $D(\ell), \ell \in \boldsymbol{L}$. These are reduced to the block-diagonal matrices, with irreducible representations $D^{(\mu)}(\ell)$ (of the dimension $|\mu|$, each appearing $f_{\mu}$ times) on the diagonal in the symmetry adapted basis $(\mathrm{SAB})$. Such a basis $\left|\mu t_{\mu} m\right\rangle(m=1, \ldots,|\mu|)$ satisfies:

$$
\forall \ell \in \boldsymbol{L}: \quad D(\ell)\left|\mu t_{\mu} m\right\rangle=\sum_{m^{\prime}=1}^{|\mu|} D_{m^{\prime} m}^{(\mu)}(\ell)\left|\mu t_{\mu} m^{\prime}\right\rangle, \quad t_{\mu}=1, \ldots, f_{\mu} .
$$

As the Hamiltonian commutes with each $D(\ell)$ there is a SAB also being the eigenbasis of the Hamiltonian. Thus, the symmetry enables us to solve the Hamiltonian eigenequation by parts: each irreducible representation singles out one $f_{\mu}$ dimensional eigenequation. In the case of finite groups, this reduction is realized by the Wigner group projectors [16]. However, for crystalline 


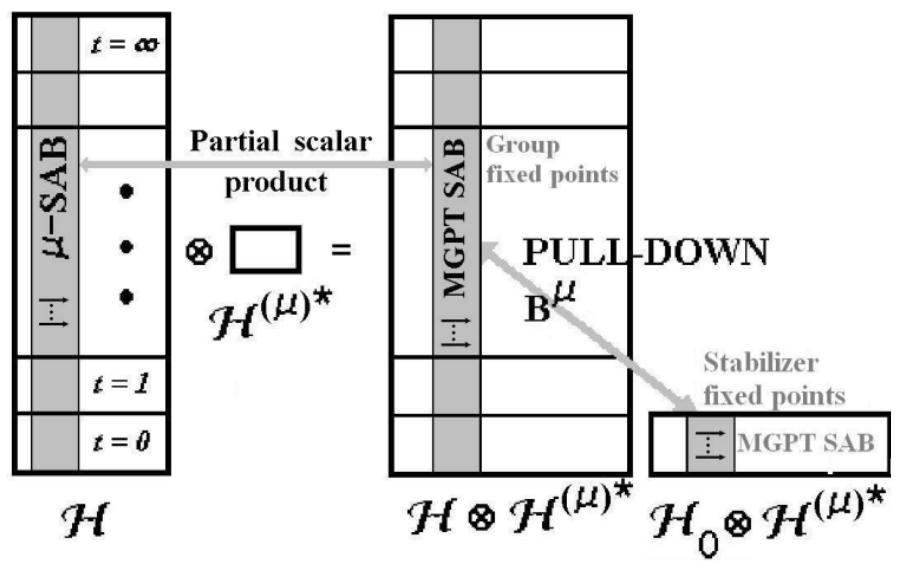

Fig. 2.4. Scheme of the modified group projector technique in the induced spaces

systems, due to the infinite symmetry group and the infinite dimensional state, this procedure, involving summation of $D(\ell)$ over group elements, is inappropriate at least numerically. These deficiencies are cured by the modified group projector technique (MGPT) $[17,18]$. It is fully implemented into the polymer symmetry simulation package POLSym devoted to the physics of the quasi-1D crystals [19], which is used in the forthcoming calculations.

The corner stone of MGPT is simple: in the space $\mathcal{H} \otimes \mathcal{H}^{(\mu)^{*}}$ (here $\mathcal{H}^{(\mu)}$ is the space of the irreducible representation $D^{(\mu)}$, and the asterisk denotes its dual) the basis $\left|\mu t_{\mu}\right\rangle$ of the fixed points of the representation $D \otimes D^{(\mu)^{*}}$ generates $\mathrm{SAB}\left|\mu t_{\mu} m\right\rangle$ in $\mathcal{H}$ by the partial scalar product:

$$
\left|\mu t_{\mu} m\right\rangle=\left\langle\mu m \mid \mu t_{\mu}\right\rangle \quad(m=1, \ldots,|\mu|),
$$

where $|\mu m\rangle$ are the standard vectors (2.16) of the irreducible representation. Thus, one needs to find the set of fixed points (Fig. 2.4): this is the range of the identity representation Wigner projector $L^{\mu}$ for $D \otimes D^{(\mu)^{*}}$ (and not $D$; thus the name modified projector). Since the fixed points of the whole group coincide with the common fixed points of the group generators, common eigenvectors for the eigenvalue 1 of the group generators are found; summation over the group is avoided.

The infinite dimension of the state space is overcome with the help of symcell and the convenient properties of $L^{\mu}$. The action of the group $\boldsymbol{L}$ on a single atom gives a set of atoms called orbit. This way, the whole compound decomposes into disjoint orbits. Taking from each orbit $O_{P}$ an arbitrary atom $P$ (orbit representative), one gets the symcell. Further, all the transformations of $\boldsymbol{L}$ leaving $P$ invariant form a finite subgroup $\boldsymbol{L}_{P}$, called stabilizer of $P$, which induces the partition of $\boldsymbol{L}$ onto the cosets: $\boldsymbol{L}=\boldsymbol{L}_{P}+z_{1} \boldsymbol{L}_{P}+\ldots$ The coset representatives form transversal $\boldsymbol{Z}_{P}=\left\{z_{0}=e, z_{1}, \ldots\right\}$. Obviously, the 
complete orbit $O_{P}$ is generated from the orbit representative by the action of $\boldsymbol{Z}_{P}$.

We consider the case when all the transversals are the same, $Z_{P}=\boldsymbol{Z}$; otherwise, one can use the intersection of all the transversals; this enlarges the set of orbit representatives and the dimension of the eigenvalue problem. In the single-particle models, such as the tight-binding one, the total state space $\mathcal{H}$ is decomposed as the sum $\mathcal{H}=\sum_{t} D\left(z_{t}\right) \mathcal{H}_{0}$ of the finite dimensional isomorphic subspaces generated by the symmetry operations from the symcell space $\mathcal{H}_{0}$. In fact, in the electron (phonon) band calculations each symcell atom contributes by some number of orbitals (Cartesian basis), spanning the atomic space $\mathcal{H}_{0 P}$, and the symcell space is the sum of these: $\mathcal{H}_{0}=\sum_{P} \mathcal{H}_{0 P}$. As the group action does not mix the orbits, the representation $D(\boldsymbol{L})$ is decomposed into the orbit subrepresentations $D(\boldsymbol{L})=\sum_{P} D_{P}(\boldsymbol{L})$, each of them being induced from a representation of $\boldsymbol{L}_{P}$ by the transversal $\boldsymbol{Z}_{P}$. Therefore, in each of the spaces $\mathcal{H}_{0 P}$ acts the representation $D_{P}^{\downarrow}$, the representation $D_{P}$ of $\boldsymbol{L}$ being restricted to the stabilizer $\boldsymbol{L}_{P}$. Thus, for each irreducible representation of $\boldsymbol{L}$, one finds the representation of the stabilizer: $\gamma^{\mu}(s)=\sum_{P} D_{P}^{\downarrow}(s) \otimes D^{(\mu)^{*}}(s)$ (for each $s$ from $\boldsymbol{L}_{P}$ ), defined in the finite dimensional product $\mathcal{H}_{0} \otimes \mathcal{H}^{(\mu)^{*}}$. The main fact is that the fixed point subspace of $D \otimes D^{(\mu)^{*}}$ in $\mathcal{H} \otimes \mathcal{H}^{(\mu)^{*}}$ is isometric [17] to the fixed points of $\gamma^{\mu}$ in $\mathcal{H}_{0} \otimes \mathcal{H}^{(\mu)^{*}}$. One has to find a modified Wigner projector for $\gamma^{\mu}$, and any basis $\left|0 ; \mu t_{\mu}\right\rangle$ in its range. Only a finite number of symmetry elements (stabilizer or site symmetry group) and finite dimensional representations are used!

The basis $\left|\mu t_{\mu} m\right\rangle$ is achieved by (2.17), where the basis of the fixed points $\left|\mu t_{\mu}\right\rangle$ is generated from the symcell space basis $\left|0 ; \mu t_{\mu}\right\rangle$ found with help of the mentioned partial isometry $B^{\mu}$ ( $c$ is a positive number making this operator isometry on $\left.\mathcal{H}_{0} \otimes \mathcal{H}^{(\mu)^{*}}\right)$ :

$$
\left|\mu t_{\mu}\right\rangle=B^{\mu}\left|0 ; \mu t_{\mu}\right\rangle, \quad B^{\mu}=c \sum_{P} B_{P}^{\mu}, \quad B_{P}^{\mu}=\sum_{t} \mathbf{1}_{0 P} \otimes D^{(\mu)^{*}}\left(z_{t}\right) .
$$

Note that the vectors $\left|\mu t_{\mu}\right\rangle$ are given by the components in the atomic basis. Their components on atoms obtained from symcell by the action of the transversal element $z_{t}$ are found as the terms (2.18):

$$
\left|\mu t_{\mu}\right\rangle^{t}=c \sum_{m} D_{m m^{\prime}}^{(\mu)^{*}}\left(z_{t}\right)\left|\mu t_{\mu} m\right\rangle^{0}, \quad\left|\mu t_{\mu} m\right\rangle^{0}=\left\langle\mu m \mid 0 ; \mu t_{\mu}\right\rangle .
$$

Finally, to find $\mathrm{SAB}$ as the eigenbasis for $H$, in the same space $\mathcal{H}_{0} \otimes \mathcal{H}^{(\mu)^{*}}$ the pulled-down Hamiltonian is used:

$$
H^{\downarrow \mu}=\sum_{P} B_{P}^{\mu^{\dagger}} H B_{P}^{\mu}
$$

It commutes with all $\gamma^{\mu}(s)$, and its eigenvectors, which are simultaneously the fixed points of $\gamma^{\mu}$, generate the $\mu$-th multiplet of SAB in $\mathcal{H}$ through (2.18) and (2.17). The corresponding eigenenergies of $H$ and $H^{\downarrow \mu}$ coincide [17]. 
The procedure must be performed for each irreducible representation of $\boldsymbol{L}$, and therefore the energies obtained are automatically assigned by the irreducible representation, i.e. by the complete set of the quantum numbers. The dimension of the eigenproblem in each step is the product of the dimension of the irreducible representation (the degeneracy of the eigenvalues obtained) and the dimension of the symcell space (the number of included orbitals for electrons and trice the number of atoms for phonons). When $\boldsymbol{L}$ describes pure translational symmetry, the algorithm reduces to the Bloch theorem, showing that MGPT generalizes it maximally, i.e. to the full symmetry group.

\subsubsection{Symmetry and Band Topology}

Some general properties of electron and phonon bands are deduced directly from the irreducible representations of the symmetry group, since Hamiltonian eigenvalues are neatly related to them.

Roto-translational elements of the line groups [20] form the abelian subgroup $\boldsymbol{T}_{q}^{r}(a) \boldsymbol{C}_{n}$, with one-dimensional representations

$$
{ }_{k} A_{m}\left(\ell_{t s 0}\right)=\mathrm{e}^{\mathrm{i} \psi_{m}^{k}(t, s)}, \quad \text { where } \psi_{m}^{k}(t, s)=\frac{k a n+2 \pi m r}{q} t+\frac{2 \pi m}{n} s
$$

defined by the pair $\mu=(k, m)$ of quasi momentum (along the $z$-axis) $k \in$ $(-\pi / a, \pi / a]$ and quasi-angular momentum $(z$ component) $m \in(-q / 2, q / 2]$. Accordingly, the corresponding symmetry adapted Bloch states in the state space are $\left|k m t_{k m}\right\rangle$. Besides these transformations, the NT symmetry group contains $U=\ell_{001}$, reversing both momenta; consequently, $U\left|k m t_{k m}\right\rangle=\left|-k,-m t_{k, m}\right\rangle$. Only when $k=0, \pi / a$ and simultaneously $m$ takes very special values (e.g. for $k=0$ and $m=0, q / 2$ ) vectors $\left|k m t_{k m}\right\rangle$ and $\left|-k,-m t_{k, m}\right\rangle$ are either the same or of the opposite sign. Consequently, half $k \in[0, \pi / a]$ of the Brillouin zone suffices to count different representations, and the pairs ${ }_{k} A_{m}$ and ${ }_{-k} A_{-m}$ are joined into double degenerate representations ${ }_{k} E_{m}$. Only the special pairs $(k, m)$ are unaffected by $U$, giving even and odd pairs with respect to $U$ one dimensional representations (e.g. ${ }_{0} A_{0}^{ \pm}$, ${ }_{0} A_{q / 2}^{ \pm}$). The corresponding $\mathrm{SAB}$ becomes $\left|k m \Pi_{U} ; t_{k m \Pi_{U}}\right\rangle$, where the parity $\Pi_{U}= \pm$ for the special representations. Achiral tube representations are four-dimensional ${ }_{k} G_{m}$ in general, since additionally $\sigma_{\mathrm{v}}$ changes the sign of $m$ and $\sigma_{\mathrm{h}}$ that of $k$; still, as $\sigma_{\mathrm{h}}$ leaves the invariant $m=0, n$ bands, and $\sigma_{\mathrm{h}}$ energies at $k=0, \pi / a$, these special representations are one- or two-dimensional, obtaining corresponding parities. The parity with respect to the horizontal (vertical) mirror plane is denoted as $\Pi_{\mathrm{h}}= \pm\left(\Pi_{\mathrm{v}}=A / B\right)$. Therefore, the label of representation has the general form ${ }_{k} \Gamma_{m}^{\Pi}$, where $\Pi= \pm$ for $U$ or $\sigma_{\mathrm{h}}$ even or odd representations (no superscript when neither of the two parities is defined), and $\Gamma$ shows the dimension: $A$ (or $B$ for $\sigma_{\mathrm{v}}$ odd representations) $E$ and $G$ for one-, two- and four-dimensional representations. Frequently it is convenient to use parity quantum numbers $\Pi= \pm 1$ and $\Pi=0$ for even/odd states and without specific parity. 
This analysis shows that the energy bands of NTs are at least two-fold [21]. Indeed, ignoring at first $U$ symmetry, one obtains bands assigned by the quantum numbers $k m$ of the subgroup $\boldsymbol{T}_{q}^{r}(a) \boldsymbol{C}_{n}$ : one band $\epsilon_{m}(k)$ along the full Brillouin zone for each $t_{k m}=1, \ldots, f_{k m}$. Then, a posteriori interrelating pairs of the Bloch states by $U$ symmetry, one finds the degeneracy $\epsilon_{m}(k)=$ $\epsilon_{-m}(-k)$ of the eigenenergies. In achiral cases bands are four-fold, except those with $m=0, n$ which are double degenerate and the corresponding Bloch functions are either even or odd in $\sigma_{\mathrm{v}}$.

Note that $U$ and $\sigma_{\mathrm{h}}$ even and odd representations may appear only at the band edges, for $k=0, \pi / a$. The corresponding states are Van Hove singularities. Indeed, for example for the band $\epsilon_{0}(k)$, either $U$ or $\sigma_{\mathrm{h}}$ symmetry implies $\epsilon_{0}(k)=\epsilon_{0}(-k)$, and such an even function of $k$ is extremal at $k=0$. Therefore, in achiral SWNTs all bands end up by Van Hove singularities at $k=0$, and in chiral ones only the bands with $m=0, q / 2$. For chiral SWNTs $q$ is quite large (at least 14 for $(4,1)$, but typically more than one hundred) and these systematic density of states (DOS) singularities are rare. On the contrary, in double-wall tubes $Q$ is at most 14, making singularities much more frequent; in fact, $Q$ is usually one or two when all the bands end up at $k=0$ by DOS singularity.

Also, the dimension of the symcell space significantly differs in singleand double-wall tubes. The symcell of any single-wall carbon nanotube contains a single atom, making the eigenproblem for the phonon spectra at most 12-dimensional, while the electronic eigenproblem is at most four- and 16dimensional in $p^{\perp}$ - and $s p^{3}$-orbital model, respectively. On the other hand, the number of atoms in the symcell of double-wall carbon nanotubes varies from three (in the most symmetric tubes) to several hundreds when the translational symmetry and $U$-axis only are present.

\subsubsection{Quantum Numbers and Selection Rules}

Quantum mechanical description of the physical processes of NTs is inevitably related to the conservation involved laws. In general, a perturbation $Q_{k m \Pi}$ with well defined quantum numbers $k, m$ and $\Pi$ ( $\Pi$ stands for all appearing parities) induces interband transitions of electrons and creation/annihilation of phonons in NT. The behavior of NT is determined by the transition probabilities, being the squares of absolute values of the matrix elements $\left\langle k_{\mathrm{f}} m_{\mathrm{f}} \Pi_{\mathrm{f}} ; t_{\mathrm{i}}\left|Q_{k m \Pi}\right| k_{\mathrm{i}} m_{\mathrm{i}} \Pi_{\mathrm{i}} ; t_{\mathrm{i}}\right\rangle$ (initial and final states are labelled as $\mathrm{i}$ and $\mathrm{f}$ ). Therefore, Clebsch-Gordan coefficients of NT symmetry groups [21], as proportional to these matrix elements (by the Wigner-Eckart theorem), give the most detailed information on the conservation laws. The selection rules, singling out the allowed transitions, are their comprehensive form. Note that assignation of bands and Bloch functions of NT by the complete set of quantum numbers is inevitable for application of the selection rules.

For the vertical interband transitions induced by a perturbation with $k=0$, the quasi momentum is conserved, and the selection rules are: 
M. Damnjanović et al.

$$
\Delta k=k_{\mathrm{f}}-k_{\mathrm{i}}=0, \quad \Delta m=m_{\mathrm{f}}-m_{\mathrm{i}}=m, \quad \Pi_{\mathrm{f}} \Pi \Pi_{\mathrm{i}} \neq-1 .
$$

In the non-vertical transitions, the rules are the same, except for the Umklapp processes, when $k_{\mathrm{i}}+k$ are out of the Brillouin zone and $\Delta m$ obtains an additional term [21]. This manifests that $m$ is not a conserved quantum number: it is related to the isogonal group (2.6), also containing rotations that are not isolated (but only combined with translations) symmetries of NTs. Therefore, sometimes it is plausible to use the alternative set of conserved quantum numbers $[5,18]$. These helical quantum numbers [20] are the helical momentum $\tilde{k}$, including linear and part of the angular momentum, and the remaining angular momentum $\tilde{m}$, related to $k$ and $m$ as:

$$
\begin{aligned}
\tilde{k} & =k+m \frac{2 r \pi}{n a}+\tilde{\kappa} \frac{2 q \pi}{n a}, & \tilde{m} & =m \bmod n, \\
k & =\tilde{k}-\tilde{m} \frac{2 r \pi}{n a}+\kappa \frac{2 \pi}{a}, & m & =(\tilde{m}-\kappa p) \bmod q .
\end{aligned}
$$

The integers $\tilde{\kappa}$ and $\kappa$ are introduced to provide $\tilde{k} \in(-q \pi / n a, q \pi / n a]$ and $k \in(-\pi / a, \pi / a]$, respectively, while $p$ is the helicity $(2.4)$.

\subsubsection{Electron Bands}

In the graphene layer the bonding of the carbon atoms is realized by three inplane hybridized $s p^{2}$ orbitals, while the forth $p^{\perp}$ orbital ( $p$ state perpendicular to the plane) is responsible for the electronic properties. As mentioned before, the curvature induced rehybridization in the tubes with a diameter larger than $7 \AA$ is weak, and the $p^{\perp}$ approximation is sufficiently good. However, for narrow nanotubes, the influence of the folded geometry on the atomic orbitals is significant, and the correct dynamical model is $s p^{3}$ hybridization, including $2 s$ and three $2 p$ carbon orbitals. As already emphasized, the simply folded configuration is unstable, but the relaxed one retains the same symmetry.

In the tight-binding (TB) approach, each atom $\mathrm{C}_{t s u}$ contributes to the state space by a set of orbitals $|t s u ; \phi\rangle$ : In the $s p^{3}$ model four orbitals $(\phi=1, \ldots, 4)$ are included, while in the $p^{\perp}$-approximation $\phi=1$ denotes the $p^{\perp}$-orbital. Therefore, in the view of the single atom SWNT symcell, the pulled down Hamiltonian (2.20) takes the simple form:

$$
H_{k m \Pi}^{\downarrow}=\sum_{t s u}\left(D^{k m \Pi}\right)^{T}\left(l_{t s u}\right) \sum_{\phi \phi^{\prime}} h_{t s u}^{\phi \phi^{\prime}}|t s u, \phi\rangle\left\langle 000, \phi^{\prime}\right| \otimes \mathbf{1}_{k m \Pi} .
$$

Recall that according to MGPT the first sum only runs over the carbon atoms $\mathrm{C}_{t s u}$ interacting with the initial one $\mathrm{C}_{000}$, while the second sum is the part of the Hamiltonian describing this interaction through the potential matrix elements $h_{t s u}^{\phi \phi^{\prime}}=\left\langle 000 ; \phi|H| t s u ; \phi^{\prime}\right\rangle$. Also, the same expression describes the pulled down overlap matrix, when instead of $h_{t s u}^{\phi \phi^{\prime}}$ pure overlaps $S_{t s u}^{\phi \phi^{\prime}}=\left\langle 000 ; \phi \mid t s u ; \phi^{\prime}\right\rangle$ are used. To proceed, additional assumptions on the included orbitals and neighbors, as well as to the proposed interaction are needed. 


\section{Tight-Binding $p^{\perp}$-Orbital Model}

Within the $p^{\perp}$ approximation with overlaps of atomic orbitals neglected, substituting in (2.25) the form of the irreducible representations of the line groups [18], for each $m$ the pulled down Hamiltonian is two-dimensional [21]:

$$
H_{m}(k)=\left(\begin{array}{cc}
h_{m}^{0}(k) & h_{m}^{1 *}(k) \\
h_{m}^{1}(k) & h_{m}^{0}(k)
\end{array}\right), \quad h_{m}^{u}(k)=\sum_{t s} h_{t s u}^{11} \mathrm{e}^{\mathrm{i} \psi_{k}^{m}(t, s)}(u=0,1) .
$$

This refers to the chiral tubes, while the achiral ones can be treated as a special case with $H_{m}(k)$ and $H_{-m}(k)$ joined into a single four dimensional matrix. Solving the eigenproblem of (2.26), for each $m$ two double degenerate bands are found as discussed in Sect. 2.3.2:

$$
\epsilon_{m}^{ \pm}(k)=h_{m}^{0}(k) \pm\left|h_{m}^{1}(k)\right| .
$$

The corresponding generalized Bloch eigenfunctions are $\left(h_{m}^{k}=\operatorname{Arg}\left(h_{m}^{1}(k)\right)\right)$ :

$$
\begin{aligned}
& |k m ; \pm\rangle=\sum_{t s} \mathrm{e}^{-\mathrm{i} \psi_{m}^{k}(t, s)}\left(|t s 0\rangle \pm \mathrm{e}^{\mathrm{i} h_{m}^{k}}|t s 1\rangle\right), \\
& |-k,-m ; \pm\rangle=\sum_{t s} \mathrm{e}^{\mathrm{i} \psi_{m}^{k}(t, s)}\left(|t s 1\rangle \pm \mathrm{e}^{\mathrm{i} h_{m}^{k}}|t s 0\rangle\right) .
\end{aligned}
$$

Note again that for the achiral tubes four-fold degeneracy appears due to $\epsilon_{m}^{ \pm}(k)=\epsilon_{-m}^{ \pm}(k)$, except that the bands $\epsilon_{m}^{ \pm}(k)=\epsilon_{m}^{A / B}(k)$ for $m=0, n$ with the opposite vertical mirror parity remain double degenerate.

The simplest $p^{\perp}$ approximation completely neglects local distortions of graphene hexagons and includes only the first three neighbors pointed to by $u_{1}=u_{2}=u_{3}=1$ and

$$
\begin{aligned}
& t_{1}=-\frac{n_{2}}{n} \\
& t_{2}=\frac{n_{1}}{n}, \\
& t_{3}=t_{1}+t_{2}, \\
& s_{1}=\frac{2 n_{1}+(1+r \mathcal{R}) n_{2}}{q \mathcal{R}}, \quad s_{2}=\frac{(1-r \mathcal{R}) n_{1}+2 n_{2}}{q \mathcal{R}}, \quad s_{3}=s_{1}+s_{2} .
\end{aligned}
$$

Therefore, all the interatomic matrix elements are equal, $h_{t_{i}, s_{i}}^{11}=V \approx-3 \mathrm{eV}$, while $h_{000}^{11}=0$. The model is analytically solvable: the pairs of $m$-assigned bands are symmetrical with respect to the Fermi level:

$$
\epsilon_{E_{m}}^{ \pm}(k)= \pm\left|V \sum_{i=1}^{3} \mathrm{e}^{\mathrm{i} \psi_{m}^{k}\left(t_{i}, s_{i}\right)}\right| .
$$

According to (2.28), for the tubes with $n_{1}-n_{3}$ divisible by 3 , there are bands crossing at the Fermi level $E_{\mathrm{F}}=0$. Precisely, if $\mathcal{R}=3$, two symmetric bands with $m=n r(\bmod q)$ cross at $k=2 \pi / 3 a$, while for $\mathcal{R}=1$ two pairs of bands with $m= \pm \frac{q}{3}$ come together in $k=0$. Apparently this implies that 

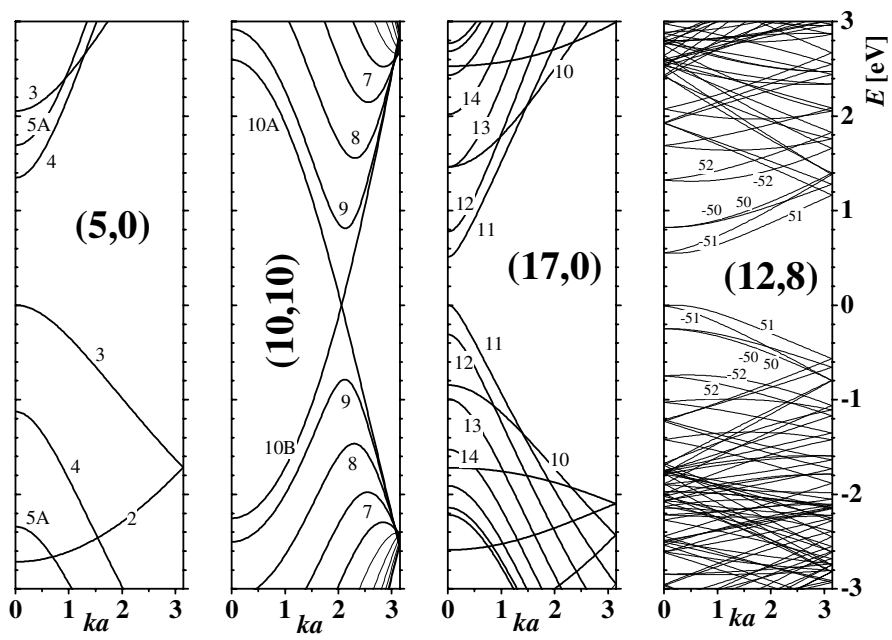

Fig. 2.5. Symmetry assigned $p^{\perp}$ orbital electronic bands of SWNTs. Note Van Hove singularities in $k=0$ for the even or odd states of the $U$-axis, as predicted in Sect. 2.3.2, and a large gap in the case of $(5,0)$. The bands are assigned by the quantum number $m$ and in the case of the achiral tubes, by a vertical mirror parity if defined ( $A$ for even and $B$ for odd). The bands of the chiral tubes are double degenerate (representations ${ }_{k} E_{m}$ ); those of the achiral tubes are four-fold (representations ${ }_{k} G_{m}$ ), except the ones with well defined vertical mirror parity (representations ${ }_{k} E_{m}^{A}$ or ${ }_{k} E_{m}^{B}$ ). The edge states are singlets or doublets

such tubes are conducting (Fig. 2.5). However, Landau's non-crossing rule forbids this: the crossing bands are assigned by the same quantum numbers, and any perturbation (such as neglected curvature effects) produces a small gap [5,22]. The exceptions are the armchair tubes: for them $q=2 n, r=1$, and $\mathcal{R}=3$, giving $m=n$, and the crossed bands differ in vertical mirror parity $[5,23]$.

Thus, this naive model together with the non-crossing rule gives the classification of SWNTs according to their conducting properties: the armchair tubes are conducting, the other tubes with integer $\left(n_{1}-n_{2}\right) / 3$ are quasimetallic with a small secondary gap less than $0.1 \mathrm{eV}$ decreasing with the tube diameter [22] as $D^{-2}$. All other tubes are semiconducting, with the gap decreasing with the tube diameter as $1 / D$; starting with more than $1 \mathrm{eV}$ for narrow tubes, the gap tends to the zero graphene limit [4]. Of course, the whole model and therefore these conclusions are not applicable to ultra narrow tubes.

The $p^{\perp}$-orbital approximation may be refined by considering the interatomic overlaps, and for thick enough tubes such results give reasonably good predictions of optical properties [24]. Also, the approximation is improved by including higher level neighbors. A model with 12 neighbors (three first, six second and three third ones, Fig. 2.2), neglecting local distortions 
and overlaps effectively uses three different constants $h_{t s u}^{11}$, one for each level of neighbors. This model, with fitted constants, describes [25] satisfactorily the bands near the Fermi level, obtained by density functional techniques.

\section{Density Functional Model}

As has been stressed, $p^{\perp}$ approximation fails to describe electronic states of narrow SWNTs: large curvature substantially affects hybridization, and all $s p^{3}$ carbon orbitals have to be considered. The band structure may be satisfactorily studied only with the help of a density functional method. According to the exchange-correlation functional employed, several types of DF methods are distinguished. All of them give similar stable configurations (Table 2.1) and predict well the conductivity, while some other details of band structure may differ significantly. The most frequently used local density approximation $[11,26,27]$ systematically overestimates the gap in semiconductors, making the predictions of optical properties not completely reliable. On the other hand, G. Seifert's [28] density functional tight-binding (DFTB) method applies the density functional technique to small molecules, and fits the Hamiltonian and overlap matrix elements of (2.25) as a functions of the interatomic distance $h^{\phi \phi^{\prime}}(r)$ and $S^{\phi \phi^{\prime}}(r)$. As these functions are well transferable to compounds with the same local structure, they can be used within the tight binding approach in studies of complex structures. Therefore, this method is more efficient (e.g. also thin SWNTs or inorganic tubes may be considered); possible deficiencies of the semiempirical foundation are at least compensated for to some extent controllable parameters (enabling to fit the experiments), and complete symmetry implementation (due to application of the DF potential through the tight-binding calculations). However, currently all DF methods fail to describe correctly Van der Waals systems; therefore, these methods are not reliable for multi-wall carbon nanotubes, despite some recent attempts to overcome this difficulty [29].

In comparison to the $p^{\perp}$ orbital results, DFTB bands of narrow tubes (see Fig. 2.6) differ substantially. In fact, the tubes $(4,0),(5,0)$ and $(4,1)$ are conductive, in contrast to the $p^{\perp}$ approximation based criterion. Of course, the symmetry based general properties of band topology, such as degeneracy and $k=0, \pi / a$ Van Hove singularities, are manifested in both models.

\subsubsection{Force Constants Phonon Dispersions}

There are lattice dynamics studies of NTs based on various force field models. Since $a b$ initio methods [30] are restricted to thin tubes, an efficient force constant method is necessary for massive calculations enlightening general NT properties [31]. To this end graphite force constants [32] are adjusted kinematically and dynamically to nanotube geometry. Each pair $(\alpha, \beta)$ of carbon atoms defines the stretching, out-of-plane and in-plane unit vectors 

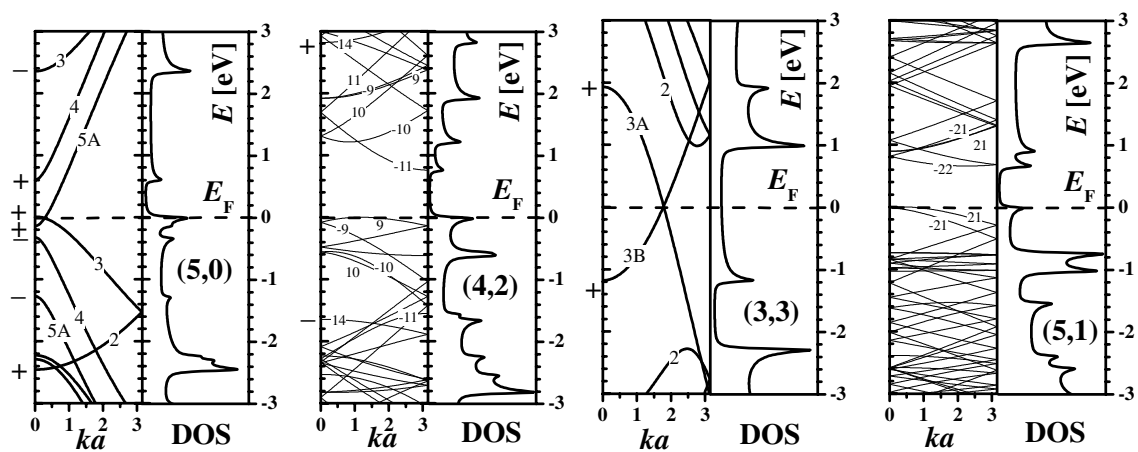

Fig. 2.6. DFTB bands and DOS of narrow tubes near Fermi level $E_{F}$ (broken horizontal line at $0 \mathrm{eV}$ ). For the tubes $(4,2)$ and $(5,1)$ the bands relevant for the calculated optical interband transitions in Sect. 2.4.2 are in bold. If defined, the $z$-reversal parity at $k=0$ and $k=\pi / a$ is indicated by \pm . Note that $(5,0)$ is conducting

$|\alpha \beta, i\rangle(i=1,2,3):|\alpha \beta, 1\rangle$ points from $\alpha$ to $\beta,|\alpha \beta, 2\rangle$ is perpendicular to $|\alpha \beta, 1\rangle$ and to the tube axis, while $|\alpha \beta, 3\rangle=|\alpha \beta, 1\rangle \times|\alpha \beta, 2\rangle$. The components of these vectors in the tube reference frame are $|\alpha \beta, 1\rangle=\left(S_{1 i}^{\alpha \beta}, S_{2 i}^{\alpha \beta}, S_{3 i}^{\alpha \beta}\right)$. These vectors and the force-constants $c_{i}^{\alpha \beta}$ are the eigenvectors and eigenvalues of the $3 \times 3$ matrix $\mathcal{D}_{\alpha \beta}$, describing the contribution of the pair $(\alpha, \beta)$ to the dynamical matrix $\mathcal{D}$ of nanotube [33]: $\mathcal{D}_{\alpha \beta}|\alpha \beta, i\rangle=c_{i}^{\alpha \beta}|\alpha \beta, i\rangle$. Therefore, $\mathcal{D}_{\alpha \beta}=\sum_{i} c_{i}^{\alpha \beta}|\alpha \beta, i\rangle\langle\alpha \beta, i|$ and its matrix elements are easily found: $\mathcal{D}_{\alpha \beta}^{i j}=\sum_{p} c_{p}^{\alpha \beta} S_{i p}^{\alpha \beta} S_{j p}^{\alpha \beta}$.

The kinematic modification provides the twisting mode exactly using the rotational sum rule [34]:

$$
\sum_{\beta}\left(R_{\alpha \beta 1} \mathcal{D}_{\alpha \beta}^{2 j}-R_{\alpha \beta 2} \mathcal{D}_{\alpha \beta}^{1 j}\right)=0, \quad \forall \alpha, \quad j=1,2,3 .
$$

The sum runs over the relevant neighbors $\beta$ of the atom $\alpha(\beta \neq \alpha)$ and $R_{\alpha \beta i}$ is the Cartesian component of the vector $\boldsymbol{R}_{\alpha \beta}=R_{\alpha \beta}|\alpha \beta, 1\rangle$ from $\alpha$ to $\beta$ in the equilibrium positions. Since $S_{32}^{\alpha \beta}=0$ because $|\alpha \beta, 2\rangle$ is orthogonal to the tube axis, (2.29) reduces to:

$$
\sum_{\beta} R_{\alpha \beta}\left(c_{2}^{\alpha \beta} S_{j 2}^{\alpha \beta} S_{33}^{\alpha \beta}-c_{3}^{\alpha \beta} S_{j 3}^{\alpha \beta} S_{32}^{\alpha \beta}\right)=0, \quad j=1,2,3 ; \forall \alpha .
$$

Thus, each atom $\alpha$ gives two constraints on out-of-plane force constants $c_{2}^{\alpha \beta}$ :

$$
\sum_{\beta} R_{\alpha \beta} c_{2}^{\alpha \beta} S_{12}^{\alpha \beta} S_{33}^{\alpha \beta}=0, \quad \sum_{\beta} R_{\alpha \beta} c_{2}^{\alpha \beta} S_{22}^{\alpha \beta} S_{33}^{\alpha \beta}=0,
$$


as SWNT is a single-orbit system, MGPT calculations are reduced to the orbit representative $\alpha=\mathrm{C}_{000}$ only, and (2.30) imposes two conditions altogether. Particularly, the force constants model [32] involves eighteen up to the fourth level neighbors; collecting all the relevant coordinate factors and force constants into the vectors $S_{k}^{\alpha}=\left(R_{\alpha 1} S_{k 2}^{\alpha 1} S_{33}^{\alpha 1}, \ldots, R_{\alpha 18} S_{k 2}^{\alpha 18} S_{33}^{\alpha 18}\right)$ and $c_{2}^{\alpha}=\left(c_{2}^{\alpha 1}, \ldots, c_{2}^{\alpha 18}\right) ;(2.30)$ becomes a pair of the orthogonality conditions $c_{2}^{\alpha} \cdot S_{k}^{\alpha}=0(k=1,2)$. These quite general conditions for any dynamical model provide the twisting acoustic mode exactly. Thus, it is natural to correct minimally the graphene force constants by projecting $c_{2}^{\alpha}$ onto the orthocomplement of the plane defined by $S_{1}^{\alpha}$ and $S_{2}^{\alpha}$.

The dynamical response to the configuration changes introduced by the folding of a graphene plane is described along the lines of [35]. In the $\alpha$-frame, in which the $z$-axis coincides with the tube axis and the $x$-axis runs through the atom $\alpha$, the eigenbasis of $\mathcal{D}_{\alpha \beta}$ is ( $\varphi$ and $\psi$ are cylindrical coordinates of $\beta$ and the angle between the horizontal $x y$-plane and the bond $\alpha-\beta$ ):

$$
\begin{aligned}
|\alpha \beta, 1\rangle & =\left(-\cos \psi \sin \frac{\varphi}{2}, \cos \psi \cos \frac{\varphi}{2}, \sin \psi\right), \\
|\alpha \beta, 2\rangle & =\left(\cos \frac{\varphi}{2}, \sin \frac{\varphi}{2}, 0\right), \\
|\alpha \beta, 3\rangle & =\left(-\sin \psi \sin \frac{\varphi}{2}, \sin \psi \cos \frac{\varphi}{2},-\cos \psi\right) .
\end{aligned}
$$

Further, consider the graphene stretching, out-of-plane and in-plane unit vectors after the folding: $\left|\alpha_{g} \beta_{g}, 1\right\rangle$ is the tangent to the projection of the bond $\alpha-\beta$ to the tube's surface, $\left|\alpha_{g} \beta_{g}, 2\right\rangle$ is perpendicular to the tube at $\alpha$, and $\left|\alpha_{g} \beta_{g}, 3\right\rangle=\left|\alpha_{g} \beta_{g}, 1\right\rangle \times\left|\alpha_{g} \beta_{g}, 2\right\rangle$. So, in the $\alpha$-frame these vectors are:

$$
\begin{aligned}
& \left|\alpha_{g} \beta_{g}, 1\right\rangle=\left(0, \frac{\cos \frac{\varphi}{2} \cos \psi}{\sqrt{1-\sin ^{2} \frac{\varphi}{2} \cos ^{2} \psi}}, \frac{\sin \psi}{\sqrt{1-\sin ^{2} \frac{\varphi}{2} \cos ^{2} \psi}}\right), \\
& \left|\alpha_{g} \beta_{g}, 2\right\rangle=(1,0,0), \\
& \left|\alpha_{g} \beta_{g}, 3\right\rangle=\left(0, \frac{\sin \psi}{\sqrt{1-\sin ^{2} \frac{\varphi}{2} \cos ^{2} \psi}},-\frac{\cos \frac{\varphi}{2} \cos \psi}{\sqrt{1-\sin ^{2} \frac{\varphi}{2} \cos ^{2} \psi}}\right) .
\end{aligned}
$$

Assuming that the forces along $\left|\alpha_{g} \beta_{g}, i\right\rangle(i=1,2,3)$ are preserved in the course of rolling, one easily finds how the required modified constants $c_{i}^{\alpha \beta}$ are related to the original graphene ones $C_{i}^{\alpha_{g} \beta_{g}}: c_{i}^{\alpha \beta}=C_{i}^{\alpha_{g} \beta_{g}} /\left|\left\langle\alpha_{g} \beta_{g}, i \mid \alpha \beta, i\right\rangle\right|$. The expansion over sine and cosine of $\varphi / 2$ yields:

$$
\begin{aligned}
c_{1}^{\alpha \beta} & =C_{1}^{\alpha_{g} \beta_{g}}\left(2-\cos \frac{\varphi}{2}\right), \\
c_{2}^{\alpha \beta} & =C_{2}^{\alpha_{g} \beta_{g}}\left(1+\sin ^{2} \psi\left(1-\cos \frac{\varphi}{2}\right)\right), \\
c_{3}^{\alpha \beta} & =C_{3}^{\alpha_{g} \beta_{g}}\left(1+\cos ^{2} \psi\left(1-\cos \frac{\varphi}{2}\right)\right) .
\end{aligned}
$$


It turns out that the dynamical and kinematical corrections each give similar SWNT force constants, and therefore the results [31] presented here, obtained by both corrections, are close to that of [35], but with exactly four acoustic modes.

\subsection{Optical Absorption}

Being both individual and bulk sensitive, the optical response measurements [36] are, together with Raman [37] and neutron scattering and electron and X-ray diffraction, widely used as a tool for diameter and chirality distribution analysis and for total SWNT yield estimations in macroscopic samples.

In this section, we evaluate numerically, using the line group theoretical methods, the optical conductivity tensor for individual SWNTs of arbitrary geometry. We use G. Seifert's [28] DFTB calculations as input for the POLSym code. The full line group symmetry of the SWNTs is taken into account (electron correlations and curvature effects are included) and the transition matrix elements of the momentum operator are, within the dipole approximation, exactly calculated from the generalized Bloch eigenfunctions.

\subsubsection{Conventional Nanotubes}

Prediction of the polarization dependent optical absorption [38] in NTs has been confirmed by optical ellipsometry [39], as well as by reflectivity measurements [40]. In addition, polarized Raman spectroscopy on fibers of aligned SWNTs, reflectance and absorption spectra and tensor invariant measurements of the Raman active modes [41] has given evidence of strongly polarized optical transitions. Anisotropy of the dielectric function has been studied in detail by S. Tasaki and coworkers [24]: a substantial optical rotatory power and circular dichroism as well as optical activity of the NT ensemble have been predicted. Also, polarized low-frequency optical spectra of SWNTs bundles, within the gradient approximation and the lowest-order isotropic model, has been calculated [42]. On the other hand, optical activity [7] and anisotropy of absorption [43] in the isolated NTs have been inferred on the line group symmetry arguments. Recently, polarized optical absorption in SWNTs has been thoroughly studied within the symmetry based approach [44].

In this subsection the polarized optical conductivity of SWNTs with diameters between $8 \AA$ and $16 \AA$ is studied. The calculations for bundled SWNTs with mean diameter $13.6 \AA$ assuming a Gaussian diameter distribution with $\Delta D=0.5 \AA$ are carried out as well.

In the relaxation-time approximation, the interband contribution (at absolute zero temperature) to the real part of the optical conductivity tensor is (we give here only the expression for the diagonal elements as, due to the symmetry of the tubes, the non-diagonal ones vanish [7]): 


$$
\mathbb{R e} \sigma_{j j}=\frac{C}{\omega} \sum\left|\left\langle k_{\mathrm{f}} m_{\mathrm{f}} \Pi_{\mathrm{f}}\left|\nabla_{j}\right| k_{\mathrm{i}} m_{\mathrm{i}} \Pi_{\mathrm{i}}\right\rangle\right|^{2} \delta(\Delta \epsilon-\hbar \omega)
$$

where $C=2 \pi e^{2} \hbar^{2} / m_{e}^{2}, \Delta \epsilon=\epsilon_{\mathrm{f}}-\epsilon_{\mathrm{i}}$ is the energy absorbed, $j$ denotes the direction of the electric field applied, while the subscripts $i$ and $f$ refer to the initial and the final state, respectively, and the summation runs over quantum numbers $k_{\mathrm{i}}, m_{\mathrm{i}}, \Pi_{\mathrm{i}}, k_{\mathrm{f}}, m_{\mathrm{f}}, \Pi_{\mathrm{f}}$ ( $\Pi$ represents all parities). As only two tensor components are independent [7], we introduce the following notation: $\sigma_{z z}=$ $\sigma_{\|}, \sigma_{x x}=\sigma_{y y}=\sigma_{\perp}$. The form of the expression used is convenient for application of the selection rules. For any orientation of the electric field, the wave vector of the Bloch electron remains essentially unchanged in the optical transition: the crystal momentum conservation law reads $\Delta k=0$.

Operator $\nabla_{\|}$is reversed upon the $U$ transformation and invariant under all the others $\left({ }_{0} A_{0}^{-}\right.$tensor of the group $\boldsymbol{L}_{\mathcal{C}}$ and $\left.\boldsymbol{L}_{\mathcal{Z} \mathcal{A}}\right)$. As for $\nabla_{x}$ and $\nabla_{y}$, it is convenient to switch to the momentum standard components $\nabla_{ \pm}=\nabla_{x} \mp \mathrm{i} \nabla_{y}$, as these carry $2 \mathrm{D}$ space of the representation ${ }_{0} E_{1}$ for $\mathcal{C}$ and ${ }_{0} E_{1}^{+}$for the achiral tubes. The dipole optical transition selection rules are now easy to deduce.

The quasi-angular momentum $m$ selection rules depend on the direction of the perturbing electric vector: if it is parallel to the tube axis (\| polarization) the rule imposes $\Delta m=0$, while if it is orthogonal onto the $z$-axis $(\perp$ polarization) $\Delta m$ should be 1 (left circular polarization) or -1 (right circular polarization) in order for the dipole optical transition to take place.

As for the parities (if defined), the one with respect to $\sigma_{\mathrm{v}}$ is to be preserved while the one with respect to the $U$ axis or to $\sigma_{\mathrm{h}}$ is to be reversed in the case of $\|$ polarization. For $\perp$ polarization it is vice versa concerning the $\sigma_{\mathrm{h}}$-parity while no restriction is imposed onto the $\sigma_{\mathrm{v}}$-parity.

Taking into account the selection rules and substituting $\sum_{k}$ by $\frac{1}{\pi} \int_{k} \mathrm{~d} k$ in (2.34) one finds:

$$
\mathbb{R e} \sigma_{\|}=\frac{C}{\omega} \sum_{m} \int_{0}^{\pi} \operatorname{Im}\left[\frac{\left|\left\langle k m ;+\left|\nabla_{\|}\right| k m ;-\right\rangle\right|^{2}}{\epsilon_{m}^{+}(k)-\epsilon_{m}^{-}(k)-\hbar \omega-i \eta}\right] \mathrm{d} k,
$$

where $|k m ; \pm\rangle$ represent the Bloch orbitals above and below the Fermi level, respectively, while $\eta=0.04 \mathrm{eV}$ is a phenomenological broadening that suppresses the height of the resonant peaks. Analogously, starting from (2.34) it is straightforward to obtain the relation for the perpendicularly polarized field:

$$
\begin{aligned}
\mathbb{R e} \sigma_{\perp} & =\frac{C}{\omega} \sum_{m} \int_{0}^{\pi} \operatorname{Im}\left[\frac{\left|\left\langle k, m+1 ;+\left|\nabla_{+}\right| k m ;-\right\rangle\right|^{2}}{\epsilon_{m+1}^{+}(k)-\epsilon_{m}^{-}(k)-\hbar \omega-i \eta}\right. \\
& \left.+\frac{\left|\left\langle k, m-1 ;+\left|\nabla_{-}\right| k m ;-\right\rangle\right|^{2}}{\epsilon_{m-1}^{+}(k)-\epsilon_{m}^{-}(k)-\hbar \omega-i \eta}\right] \mathrm{d} k .
\end{aligned}
$$

Note that for $\mathcal{A}$ tubes $\|$ transitions for $m=0, n$ are to be excluded as doubly degenerate electronic bands have different vertical mirror symmetry. 


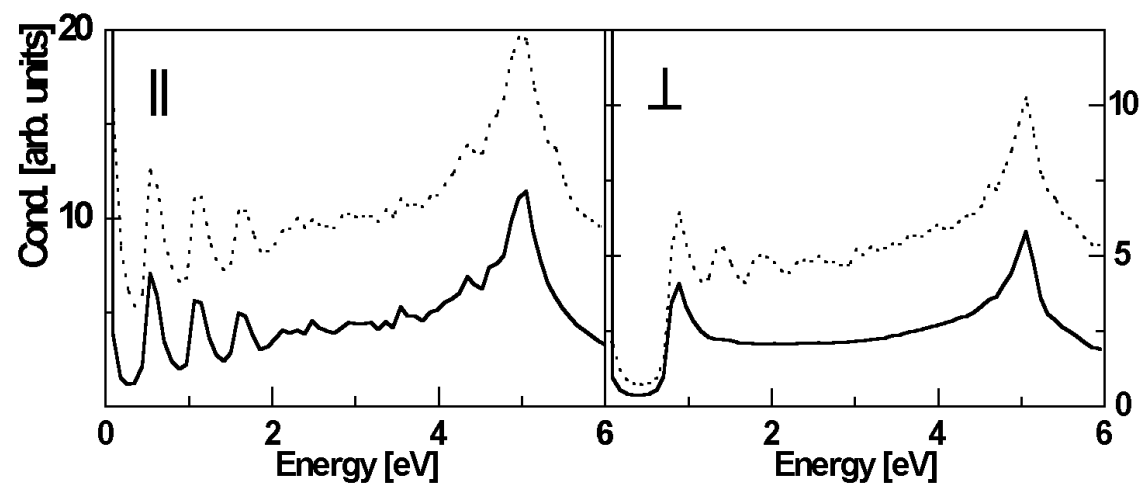

Fig. 2.7. Components of the optical conductivity tensor (solid lines) and their JDOS approximation (dotted lines) for the (10,10)-bundle. The intensity of the optical conductivity of the bundle is normalized to a single tube

Concerning the +/ - parities, they are implicitly taken into account as symmetry adapted state functions are used. Namely, in spite of the systematic strong Van Hove singularities, the $\|$ transitions between the states with nonvanishing $k$ (but close to $k=0$ ) are highly suppressed. This is due to the continuity principle, since the transitions are forbidden at $k=0$ by the $z$ reversal symmetry. As for the $\perp$ transitions, the parity selection rules are of no importance for the $\mathcal{A}$ tubes.

By contrast, for $\mathcal{Z}$ tubes the $\|$ transitions are not affected by the parities, while the $\perp$ transitions are almost entirely suppressed by them. For this type of tubes there are no DOS peaks outside the $k=0$ vicinity while the $z$-reversal symmetry forbids the $\perp$ absorption at $k=0$. Owing to the continuity of the matrix elements, the corresponding transition probabilities are small and the $\perp$ optical spectra features are highly suppressed despite the strong Van Hove singularities.

Concerning the $\mathcal{C}$ tubes, although the parities practically do not influence the optical absorption, we find that the transition probabilities are strongly helicity dependent: the results obtained differ considerably from those approximated by the joint density of states (JDOS).

A typical || polarized absorption spectrum of the bundled SWNTs (Gaussian distribution centered at $D=13.6 \AA$ with $\Delta D=0.5 \AA$ of 29 tubes comprising the $(10,10)$-bundle) is depicted in the left panel of Fig. 2.7. Note that each tube in the bundle is weighted with a Gaussian factor, i.e. the absorption intensity is normalized to a single tube. The first two peaks at $0.55 \mathrm{eV}$ and $1.1 \mathrm{eV}$ are the well-known semi-conducting tube response that scale inverse to the tube diameter. The third feature, at $1.65 \mathrm{eV}$, scaling up also with $1 / D$, comes from $\mathcal{A}$ tubes and from quasi-metallic $\mathcal{Z}$ and $\mathcal{C}$ ones.

The collective excitation of the $\pi$-electron system (at zero-momentum transfer) in the tubes with the $(10,10)$-bundle typical diameters, we find (for 


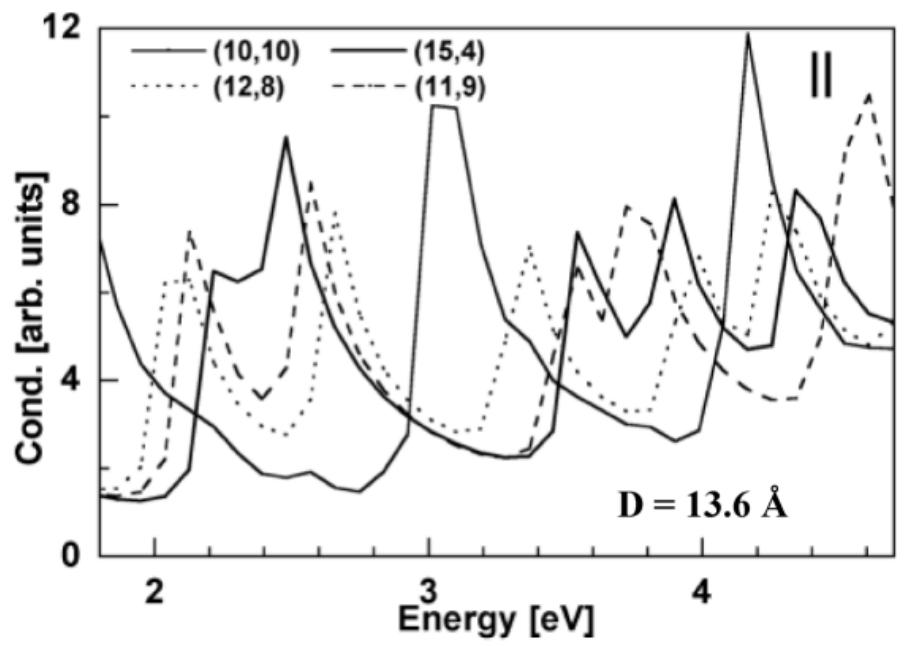

Fig. 2.8. Highly helicity sensitive \| polarized absorption spectra features (in the energy region between the well pronounced low-energy peaks and the onset of the $\pi$-plasmon) for SWNTs $(10,10),(15,4),(12,8)$ and $(11,9)$ having the same diameter $D=13.6 \AA$ but different wrapping angle

both polarizations) at $5 \mathrm{eV}$, Fig. 2.7. Considerable anisotropy is evident although the excitation is well pronounced for the $\perp$ polarized electric field as well. In the range between the low-energy inter-band transitions and the $\pi$ plasmon excitation energy few inter-band optical absorption features appear. As they are highly sensitive to the helicity of the particular tube (regarding the peak positions and absorption intensities), after averaging over a bundle they result in rather broad and weakly pronounced peaks. Their wrappingangle sensitiveness is illustrated in Fig. 2.8 by tubes with diameter $D=13.6 \AA$.

The main features of the $\perp$ polarized optical spectra are given in the right panel of Fig. 2.7. These are responses from all the types of tubes within the bundle apart from the tubes $(15,3),(16,4),(15,5),(14,5),(13,5),(14,6)$ and $(13,7)$, which are practically transparent throughout the entire range for such a polarization of the electric field.

We now turn to the inspection of the differences between the results presented here and the widely used JDOS approximated ones based on the lowest-order isotropic TB model. For \| polarization the JDOS only based results are in reasonably good agreement with more accurate calculations (Fig. 2.9), aside from the overall absorption intensity enhancement and slight differences regarding positions, relative intensities and shapes of peaks. These differences seem to be more pronounced for tubes with small wrapping angle ( $\mathcal{Z}$ tubes and tubes close to the $\mathcal{Z}$-direction) but can scarcely be noticed when averaged over a bundle. This is illustrated in the left panel of Fig. 2.7. On the contrary, in the case of the $\perp$ polarization, the JDOS approxima- 


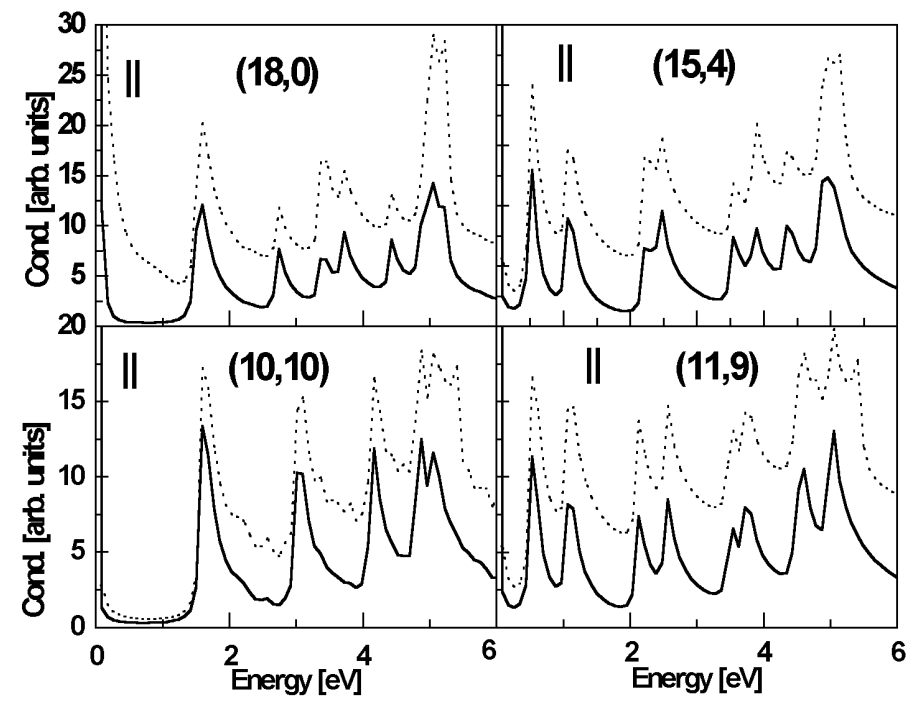

Fig. 2.9. || component of the optical conductivity (bold line) and the JDOS approximation to it (dotted line) for SWNTs $(18,0),(15,4),(10,10),(11,9)$

tion leads to incorrect predictions. As is evident from the calculated spectra for the (10,10)-bundle (Fig. 2.7, right panel), many of the absorption peaks resulting from the JDOS approximation of the $\perp$ component of the optical conductivity tensor, do not appear in the more accurately calculated optical spectrum. For isolated SWNTs these discrepancies are even more noticeable. For comparison see Fig. 2.10.

The results obtained reinforce the JDOS approximation of the optical conductivity for $\|$ polarized electric field, but lead to quite different conclusions as far as the $\perp$ component of the conductivity tensor is concerned. The discrepancies are most prominent for thin $\mathcal{Z}$ tubes, but also quite substantial even when the JDOS only based results are averaged over a bundle. We explain this by the influence of the parity selection rules on the transition probabilities, elucidating that the JDOS approximation is conceptually incompatible with the horizontal mirror parity selection rules for dipole absorption processes in achiral SWNTs. Namely, although very little weight is associated with the horizontal mirror parity states (as this parity is well defined only for a finite number of high symmetry states) due to the continuity principle (which cannot be included within the JDOS approximation), if the transition is not permitted at $k=0$ the transitions between the nearby states with general, nonvanishing $k$ are not very likely to occur. As all the bands of the achiral tubes are zero sloped at $k=0$, this means that the JDOS approximation [43] to the optical absorption spectrum of the achiral NT cannot be entirely reliable. Especially not for the $\mathcal{Z}$ tubes as all their DOS Van Hove singularities show up in the $k=0$ vicinity. In general, horizontal mirror 


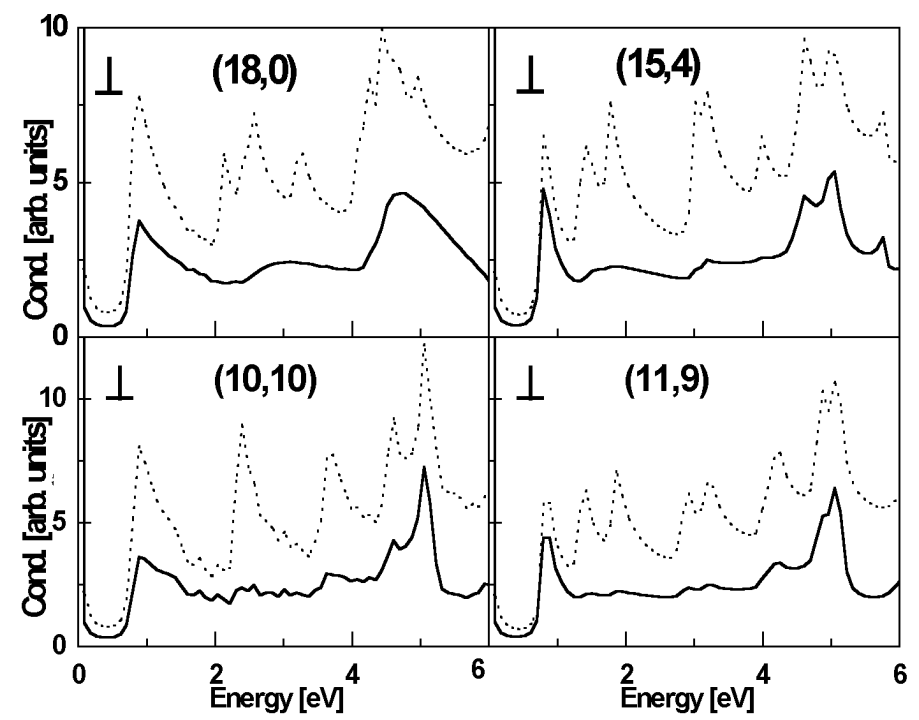

Fig. 2.10. $\perp$ component of the optical conductivity (bold line) and the JDOS approximation to it (dotted line) for SWNTs $(18,0),(15,4),(10,10),(11,9)$

symmetry suppresses a vast number (all but two) of the perpendicularly polarized optical transitions in the $\mathcal{Z}$ tubes and all the parallel transitions close to $k=0$ in the $\mathcal{A}$ tubes. Since the number of bands enlarges with diameter and they become zero sloped over larger $k=0$ vicinity, the horizontal mirror parity influence on the interband transitions is diminished for thicker tubes. In contrast, the $U$ parity does not affect the optical transitions in the chiral SWNTs.

\subsubsection{Template Grown Nanotubes}

Soon after the discovery of carbon nanotubes, the existence of narrow tubes was predicted [45]. Recent calculations confirmed [11] that SWNTs that have been synthesized inside channels of zeolite $\mathrm{AlPO}_{4}$ (AFI) crystal [46], if isolated, should be energetically stable. The diameter of the zeolite-grown tubes is determined to be $4.2 \pm 0.2 \AA$, while $7.3 \AA$ is the inner diameter of the channels of the AFI crystal, adjacent centers of which are $13.7 \AA$ distant $[47,48]$. Such a highly defined geometry of the sample makes it easier to carry out the measurements of the electro-optical properties of the zeolite-grown tubes and gives measurements closer to the theoretical models. On the basis of the optical absorption measurements and ab initio electronic band structure calculations, the NTs $(5,0),(3,3)$ and $(4,2)$ were suggested to be zeolite-grown [47]. More recently, several full DFLD calculations of the optical properties of these three tubes have been reported $[26,27,49]$. Quite recently symmetry based calculations of the optical absorption in narrow NTs has been performed [50]. 
Here we study the polarized optical absorption of NTs $(5,0),(4,2),(3,3)$ and $(5,1)$, in energy region $0-6 \mathrm{eV}$. The method applied is conceptually the same as the one described in the previous subsection where the SWNTs that are thicker than icosahedral $\mathrm{C}_{60}$ are treated. Only here, instead a single $2 p^{\perp}$ orbital (per carbon atom), we take the basis set $\left\{2 s, 2 p_{x}, 2 p_{y}, 2 p^{\perp}\right\}$, thus taking into account $\sigma-\pi$ hybridization. Also, we adopt the symmetry maintaining DFTB optimized configuration given in Table 2.1. An analogous optimization procedure was originally suggested by Cabria et al. [11], and we have used those results in [50]. This gives us an opportunity to compare the results of the full DFLD $[11,26,27,49]$ and the combined DFLD/DFTB [50] approaches to the full DFTB results here presented.

The considered SWNTs are of uniform thickness while their symmetry and chirality are quite diverse. As for the achiral NTs, symmetry of the zig-zag one, $(5,0)$, is described by the line group $\boldsymbol{L} 10_{5} / \mathrm{mcm}$, which is a product of the screw axis $\boldsymbol{T}_{10}^{1}$ and the point group $\boldsymbol{D}_{5 \mathrm{~h}}$. The isogonal point group is $\boldsymbol{D}_{10 \mathrm{~h}}$ and it is not a subgroup of the symmetry group $\boldsymbol{L} 10_{5} / \mathrm{mcm}$. (Recall that SWNT symmetry groups are non-symmorphic [7].) Line group $\boldsymbol{L} 6_{3} / \mathrm{mcm}=\boldsymbol{T}_{6}^{1} \boldsymbol{D}_{3 \mathrm{~h}}$ describes the symmetry of the armchair tube $(3,3)$, while $\boldsymbol{D}_{6 \mathrm{~h}}$ is the isogonal point group. Concerning the remaining two chiral tubes, $(4,2)$ and $(5,1)$, the screw axis group of each tube is $\boldsymbol{T}_{28}^{9}$ and $\boldsymbol{T}_{62}^{51}$. Thus, the isogonal point groups are $\boldsymbol{D}_{28}$ and $\boldsymbol{D}_{62}$, while the symmetry groups are $\boldsymbol{L} 28_{22} 22=\boldsymbol{T}_{28}^{9} \boldsymbol{D}_{2}$ and $\boldsymbol{L} 62_{45} 22=\boldsymbol{T}_{62}^{51} \boldsymbol{D}_{1}$.

In Fig. 2.6 the calculated electronic dispersions and the corresponding state density are depicted. The achiral tubes are found to be metallic, while $(4,2)$ and $(5,1)$ are indirect gap semiconductors $(\sim 0.76 \mathrm{eV}$ and $\sim 0.66 \mathrm{eV}$, respectively). Due to the $\sigma^{*}-\pi^{*}$ hybridization the double-degenerate $m=5$ electronic band with well defined vertical mirror parity (Fig. 2.6a) is lowered below the Fermi level, converting the $(5,0)$ tube from a semiconductor (within a simple TB method that includes nearest-neighbor $2 p^{\perp}-2 p^{\perp}$ interaction only) to a metal. Interestingly, the electronic DOS at the Fermi level in the $(5,0)$ turns out to be larger than that in the $(3,3) \mathrm{NT}$.

In Fig. 2.11, the optical conductivity spectra for the light polarized parallelly to the NT axis is depicted. There are two strong peaks at $1 \mathrm{eV}$ and $2.4 \mathrm{eV}$ and a weaker one at $1.5 \mathrm{eV}$ for the tube $(5,0)$. These peaks are due to the inter-band transitions (Fig. 2.6a): ${ }_{k} G_{4} \rightarrow{ }_{k} G_{4},{ }_{k} E_{5}^{A} \rightarrow{ }_{k} E_{5}^{A}$ and ${ }_{k} G_{3} \rightarrow{ }_{k} G_{3}$. Here ${ }_{k} G_{m}$ and ${ }_{k} E_{m}^{A}$ denote four-fold and double degenerate electronic bands (below and above the Fermi level) which, at $k=0$, end in double-degenerate states (transforming as $\left.{ }_{0} E_{m}^{ \pm}\right)$and non-degenerate states $\left({ }_{0} A_{m}^{ \pm}\right)$, respectively. Thus, the transitions at the $\Gamma$ point are ${ }_{0} E_{4}^{-} \rightarrow{ }_{0} E_{4}^{+}$and ${ }_{0} E_{3}^{+} \rightarrow{ }_{0} E_{3}^{-}$in the line group notation or $E_{4 \mathrm{u}} \rightarrow E_{4 \mathrm{~g}}$ and $E_{3 \mathrm{u}} \rightarrow E_{3 \mathrm{~g}}$ in the isogonal point group notation. Since the polar vector along the tube axis transforms as the ${ }_{0} A_{0}^{-}\left(A_{2 \mathrm{u}}\right)$ tensor, the parities of the edge states favor these transitions [44]. Consequently, intense absorption at these particular energies is expected. On the other hand, edge states of both ${ }_{k} E_{5}^{A}$ bands, ${ }_{0} A_{5}^{-}$and ${ }_{0} A_{5}^{+}$(i.e. $B_{2}$ and 


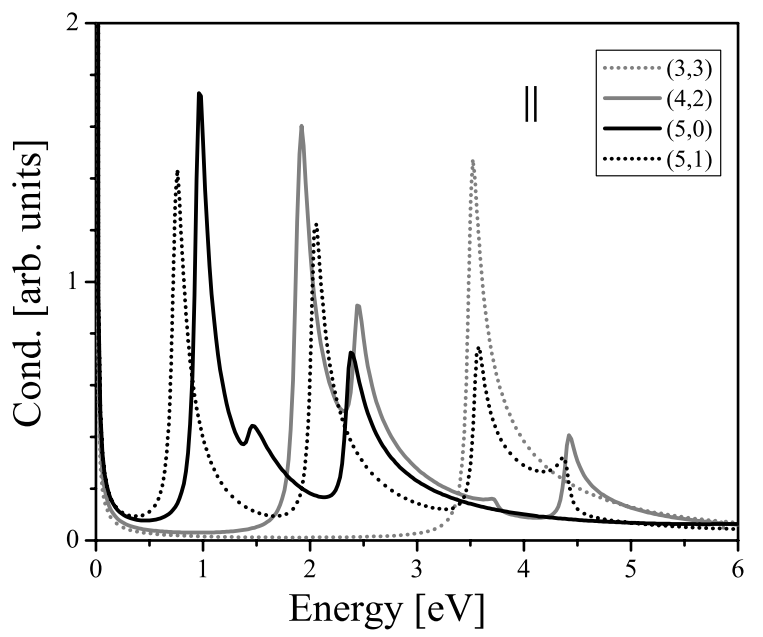

Fig. 2.11. Real part of the optical conductivity for incoming light polarized parallel to the NT axis

$\left.B_{1}\right)$, fall below the Fermi level, Fig. 2.6. The peak at $2 \mathrm{eV}$ is due to the $(5,1)$ tube inter-band transitions ${ }_{k} E_{21} \rightarrow{ }_{k} E_{21}$ (Fig. 2.6). The peaks beyond the visible absorptions, at $3.5 \mathrm{eV}$, at $4.5 \mathrm{eV}$ and at $4.4 \mathrm{eV}$ come from the tubes $(3,3),(4,2)$ and $(5,1)$ due to the dipole transitions ${ }_{k} G_{2} \rightarrow{ }_{k} G_{2},{ }_{k} E_{3} \rightarrow{ }_{k} E_{3}$, and ${ }_{k} E_{31} \rightarrow{ }_{k} E_{31}$, respectively. The tube $(4,2)$ also absorbs at $1.9 \mathrm{eV}$ and at $2.5 \mathrm{eV}$. These peaks are attributed to the transitions: ${ }_{k} E_{-10} \rightarrow{ }_{k} E_{-10}$ and ${ }_{k} E_{-11} \rightarrow{ }_{k} E_{-11}$ (Fig. 2.6b).

The absorption measurements of the tubes encased in a porous zeolite [47] show a sharp peak at $1.37 \mathrm{eV}$ with a shoulder at $1.19 \mathrm{eV}$ and two broad bands centered at $2.1 \mathrm{eV}$ and $3.1 \mathrm{eV}$. Although zeolite $\mathrm{AlPO}_{4}$ is transparent from the near infrared to the ultraviolet, it might affect the electro-optical properties of the NTs inside its channels. Recent full DFT calculations [49] show that generally, the AFI crystal has a real effect on the electronic structure and optical absorption spectra of the NTs inside its channels (some of the peaks were blue-shifted and some red-shifted after tube-crystal interaction was included). Also, many-electron effects are included only through the DFTB input data, thus not taking into account influence of the excitonic effects [51] on the optical properties of NTs. Hence, only a qualitative comparison of our results of the experiment can be made. We find the calculated intense lowest energy optical transition, due to $(5,0)$, at $1 \mathrm{eV}$, while the shoulder below it can be attributed to the transition in $(5,1)$ (Note that the diameter of the $(5,1)$ tube in the ideal cylindrical structure is $4.36 \AA$.). The first broad band can be interpreted by a convolution of the calculated absorption peaks: $1.9 \mathrm{eV}, 2.5 \mathrm{eV}$ of $(4,2), 2 \mathrm{eV}$ of $(5,1)$ and $2.4 \mathrm{eV}$ of $(5,0)$. The second broad band might correspond to the convolution of the $(3,3)$ and $(5,1)$ tube optical response at $3.5 \mathrm{eV}$. 
Table 2.3. Representations of the acoustic ( $\mathrm{T}, \mathrm{L}$ and $\mathrm{W}$ for transversal, longitudinal and twisting), radial-breathing $(\mathrm{R})$, high-energy $(\mathrm{H})$, IR and Raman active modes of SWNTs. IR and Raman activities are indicated by $\mathrm{R}$ and $I$, with the superscripts showing corresponding polarization of incident (and scattered) light. Contribution to the symmetric and antisymmetric part of the Raman tensor is emphasized by $[R]$ and $\{R\}$. The total number of IR and Raman active-modes assigned by the representation is on the right.

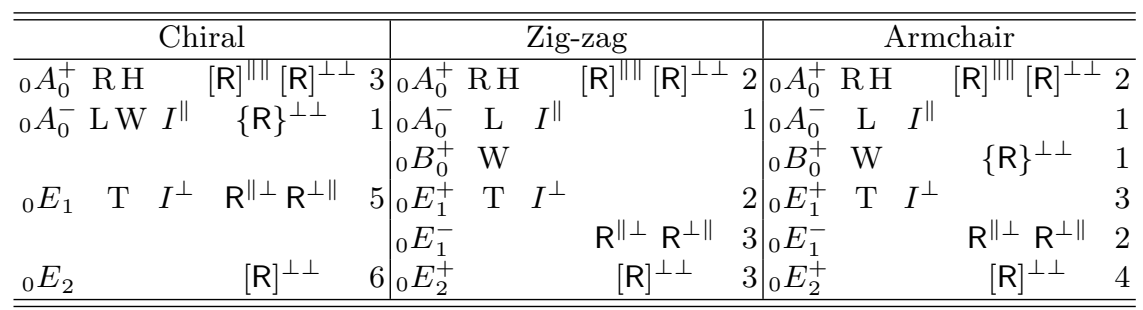

\subsection{Phonons}

Along the lines prescribed in Sect. 2.3.1 the full symmetry implemented calculation of phonon dispersions within described dynamical models is carried out for single- and double-wall NTs of various chiralities and diameters. This enables one to study various related properties, among which sound velocity, specific heat and optical scattering are selected for their comparability with standard experiments. In general, in each of these analyses the underlying physical process singles out some characteristic vibrational modes.

\subsubsection{Infinite SWNTs}

The dynamical matrix $\mathcal{D}$ is constructed from the adjusted graphene force constants as it discussed in Sect. 2.3.5. It takes the role of the Hamiltonian, and within MGPT it is easily diagonalized, due to the fact that the symcell contains a single atom, making the dimension of the eigenproblem at most 12 . The symmetry is in this case represented by the dynamical representation $D$; its decomposition into irreducible components [7] shows the number of modes assigned by any set of quantum numbers. This gives a quick, although very rough, insight to Raman [53], infra red (IR) or other properties. In Table 2.3 the quantum numbers of the relevant modes are given as the labels of the corresponding irreducible representations.

\section{Acoustic Branches: Sound Velocity and Heat Capacity}

The acoustic modes, being rigid body translations and coaxial twisting of SWNT, transform like polar vector and axial angular momentum under the symmetry operations. Longitudinal acoustic (LA) and twisting (TW) modes are nondegenerate, with the same momenta quantum numbers $k=m=0$ 

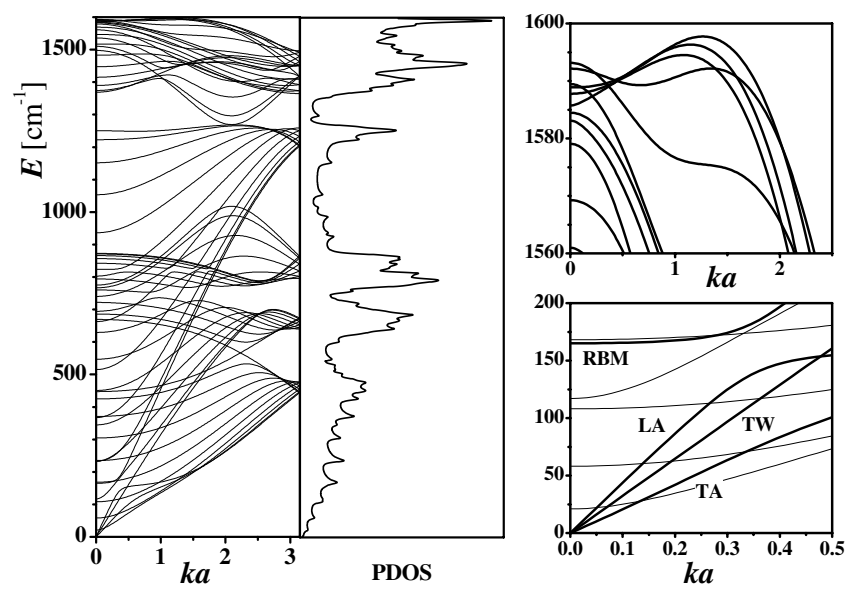

Fig. 2.12. Left: phonon dispersions and the corresponding density of states of the $(5,5)$ tube. Right: high energy bands with overbending (upper panel) and, in the lower panel, acoustic and radial breathing branches in the $\Gamma$ point region

and $U$ parity $\Pi_{U}=-1$; they differ only in the achiral tubes due to the mirror planes: $\Pi_{\mathrm{h}}^{\mathrm{LA}}=-\Pi_{\mathrm{h}}^{\mathrm{TW}}=-1$ and $\Pi_{\mathrm{v}}^{\mathrm{LA}}=-\Pi_{\mathrm{v}}^{\mathrm{TW}}=+1$. On the other hand, the $x$ and $y$ components, combined by the rotations of SWNT, give two degenerate transversal acoustic (TA) modes, characterized by $k=0$. Instead of the Cartesian components, SAB is formed by the linear combinations corresponding to $m= \pm 1$ : e.g. for linear momentum one gets $p_{ \pm}=\left(p_{x} \mp \mathrm{i} p_{y}\right) / 2$. As for the parities, $\Pi_{U}=\Pi_{\mathrm{v}}=0$ and $\Pi_{\mathrm{h}}=+1$.

All the acoustic branches are linear in $k$ near the $\Gamma$ point (Fig. 2.12), with the almost tube independent slopes equal to the sound velocities $[31,32,35]$ : $v_{\mathrm{TA}}=9.41 \mathrm{~km} / \mathrm{s}, v_{\mathrm{LA}}=20.37 \mathrm{~km} / \mathrm{s}, v_{\mathrm{TW}}=14.98 \mathrm{~km} / \mathrm{s}$. The analogy with the graphene TA branch is used in the literature $[54,55]$ to propose quadratic $k$-dependence of the nanotube TA branch. To clarify the question connected to the specific heat, the graphene and SWNT acoustic branches are interrelated. Close to the $\Gamma$ point the TA graphene acoustic branch is quadratic, while the other two (degenerate) are linear in the wave vector, as clearly explained in [56]. In the folding procedure, the degenerated in-plane translations (graphene LA modes along and perpendicular to the chiral vector) become TW and LA acoustic modes of a SWNT. Simultaneously, the graphene TA mode obviously turns into the homogeneous oscillations of the tube diameter, i.e. the radial breathing ( $\mathrm{RB}$ ) mode. Therefore, by analogy with the graphene, in the nanotube one expects linear LA and TW and a quadratic RB branch. Indeed, as discussed in Sect. 2.3.2, the totally symmetric RB mode (thus $\Pi_{U}=+1$ ) must be of Van Hove type singularity for all the SWNTs types, which implies that the corresponding band is quadratic in $k$ near $k=0$ (see Fig. 2.12). Note that two degenerate nanotube TA modes are not related to the graphene acoustic modes. 

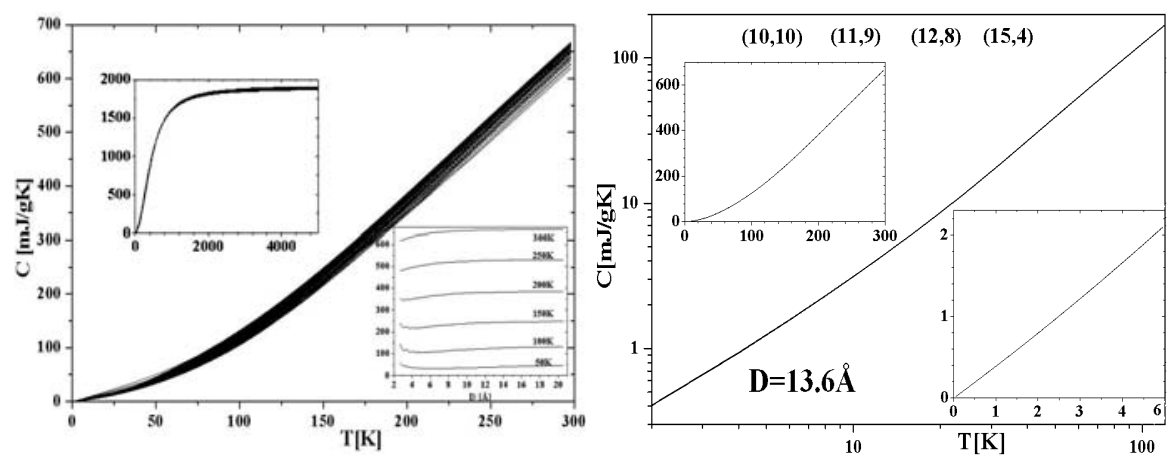

Fig. 2.13. Left: heat capacity $C_{\mathrm{ph}}$ dependence on temperature for tubes with diameters $D$ from $2.8 \AA$ to $20.5 \AA$ ( 225 tubes from $(2,2)$ to $(24,4))$. The broadening of the curve is due to the variations in $C_{\mathrm{ph}}$ with the tube diameters being depicted for various temperatures in the upper inset. The high temperature limit is visible in the lower inset. Right: heat capacities for four tubes with the same diameter $D=13.6 \AA$ coincide at all temperatures: the central (log-log plot) and upper panels show that at low temperatures $C_{\mathrm{ph}}$ is linear in $T$, the overall behavior is in the lower panel

Using phonon dispersions and densities of phonon states $[57,58]$, the specific heat of SWNTs may be calculated. Theoretical predictions of the specific heat of some particular isolated SWNT $[54,57,59]$ are in reasonably good agreement with the heat capacity measurements on nanotube bundles $[58,60,61]$. On the other hand, a fine dependence of this quantity on the nanotube parameters has been found [31] by calculations on many SWNTs in a wide range of diameters and chiralities, Fig. 2.13.

Below the temperature $T_{\mathrm{o}}=\hbar \omega_{\mathrm{min}}^{\mathrm{o}} / 6 k_{B}$, where $\omega_{\min }^{\mathrm{o}}$ is the minimal frequency of optical modes, only the acoustic modes contribute to the SWNT specific heat $[54,58]$. It is found that $T_{\mathrm{o}}$ depends on the tube diameter (in $\AA)$ as $T_{\mathrm{o}}=\left(7.2+0.05 D+1.045 D^{2}\right)^{-1} 10^{3} \mathrm{~K}$. The lowest optical branch is assigned by $m=2$. It starts at $k=0$ with Raman active modes and attains its minimum $\omega_{\text {min }}^{\mathrm{o}}$ at $k_{\mathrm{o}}$, which rapidly decreases with the tube diameter and depends on chirality (e.g. for achiral tubes $k_{\mathrm{o}}=0$ ). At low temperatures specific heat is linear in $T$; the temperature range of the linear regime gets narrower when the tube diameter increases, yielding continuous crossover to the graphene $T^{2}$ dependence.

In Fig. 2.13 the calculated specific heat temperature dependence up to $300 \mathrm{~K}$ for $225 \mathrm{SWNTs}$ is presented. It nicely matches the measured values [60]. Notable broadening of the curve with the temperature indicates slight differences in $C_{\mathrm{ph}}$ of various tubes at higher temperatures. However, this weak diameter dependence is completely saturated for considerably thin tubes (upper inset). The high-temperature limit of $3 k_{B} / m$ agrees reasonably well with the expected value for the carbon systems $[58,60]$. The chirality shows no 
signature in the specific heat of the tubes, as clearly illustrated in the right panel: the tubes $(10,10),(12,8),(15,4)$ and $(11,9)$, with the same diameter $D=13.6 \AA$ but different chiral angle, have the same specific heat.

\section{Infra Red Activity}

Infra red activity is determined by the exciting phonons in the electrical field. Within the dipole approximation [62], the perturbation operator is reduced to the projection $\boldsymbol{e} \cdot \boldsymbol{p}$ of the total ionic momentum to the polarization vector $\boldsymbol{e}$ of the incoming electric field. Consequently, linear momenta quantum numbers single out IR active modes. As discussed above $k=0$, while other quantum numbers depend on the field polarization: parallel to the tube axis field (involving $p_{z}$ momentum) $m=0, \Pi_{U}=\Pi_{\mathrm{h}}=-1, \Pi_{\mathrm{v}}=+1$, and for the perpendicular linear and circular polarizations (relating $p_{x}$ and $p_{y}$ and the standard components $\left.p_{ \pm}\right) m=1, \Pi_{U}=\Pi_{\mathrm{v}}=0, \Pi_{\mathrm{h}}=+1$. Excluding acoustic modes, for chiral tubes there are six IR active modes, out of which only one with $m=0$ is active under the parallel polarization of the light and all the others $(m=1)$ under the perpendicular (either linear or circular) polarization of the light. It is important to note that the armchair configuration has no active modes for the parallel polarization, unlike the zig-zag case where there is one such mode. Perpendicularly (or $x y$-circularly) polarized light may activate three (two) modes in the armchair (zig-zag) tubes.

\section{Raman Spectra}

In the first order Raman scattering in the incoming field $\boldsymbol{E}_{\mathrm{i}}$, exciting electrons induces in the target polarization $\boldsymbol{P}$ described by the Raman (polarizability) tensor $\mathrm{R}$ as $\boldsymbol{P}=\mathrm{R} \boldsymbol{E}_{\mathrm{i}}$. This way the ionic system is affected indirectly, i.e. phonons are created at the cost of the partial deexcitation of the electrons. The residual excitation energy is emitted as the scattered field $\boldsymbol{E}_{\mathrm{s}}$. The transition probabilities are determined by the component $\mathrm{R}_{\mathrm{is}}=\boldsymbol{e}_{\mathrm{i}} \boldsymbol{R}_{\mathrm{s}}$ of the Raman tensor corresponding to the incoming and scattered light polarizations. In general, as a second rank polar tensor, these components carry the quantum numbers $k=0, m=0, \pm 1, \pm 2$, and only the corresponding phonons participate in the scattering. Which of them will be excited depends on the polarizations $\boldsymbol{e}_{\mathrm{i}}$ and $\boldsymbol{e}_{\mathrm{s}}$, as discussed below and outlined in Table 2.3.

The most relevant symmetric Raman tensor [R] besides the isotropic (totally symmetric) component with $m=0, \Pi_{U}=\Pi_{\mathrm{v}}=\Pi_{\mathrm{h}}=+1$ includes anisotropic components with $m=1, \Pi_{U}=\Pi_{\mathrm{v}}=0, \Pi_{\mathrm{h}}=-1$ and $m=2$, $\Pi_{U}=\Pi_{\mathrm{v}}=0, \Pi_{\mathrm{h}}=+1$. Totally symmetric phonons are activated if both polarizations are parallel, $\boldsymbol{e}_{\mathrm{i}}=\boldsymbol{e}_{\mathrm{s}}$. These modes are one radial breathing, and two (chiral tubes) or one (achiral) high energy (HE) modes. Anisotropic $m=1$ modes are excited for the crossed polarizations, $\boldsymbol{e}_{\mathrm{i}} \perp \boldsymbol{e}_{\mathrm{s}}$, and $m=2$ phonons are created if the polarizations are perpendicular mutually (or left- 
and right-hand circular) and to the tube axis. Altogether, this gives 14 for chiral tubes and 8 for achiral tubes with Raman active modes $[7,53]$.

For the chiral systems [63], as well as in the resonant scattering, which is known to be important in SWNTs [64], the antisymmetric part $\{R\}$ of the Raman tensor may be important. Only one of its components is specifically antisymmetric (Table 2.3), with quantum numbers differing from those listed for $[\mathrm{R}]$; even this component is absent for zig-zag tubes. It is characterized by $m=0, \Pi_{U}=-1$ for chiral tubes, and additionally by $\Pi_{\mathrm{v}}=-1, \Pi_{\mathrm{h}}=+1$ for armchair tubes. The modes are activated by mutually parallel polarizations perpendicular to the tube. The components with $m=1, \Pi_{U}=\Pi_{\mathrm{v}}=0$, $\Pi_{\mathrm{h}}=-1$ are excited for the crossed polarizations, as well as for the symmetric components with the same assignation.

In the Raman measurements [52] on carbon nanotubes the most emphasized peaks are observed at the frequencies of the totally symmetric vibrations: besides the low energy (up to $700 \mathrm{~cm}^{-1}$ ) radial breathing mode, also a high energy (below $1600 \mathrm{~cm}^{-1}$ ) one is activated. Except for ultra narrow tubes, RB frequency is chirality independent, but directly reflects the diameter of the tube, which is widely used in the sample characterization by Raman measurements. The fit of the results for 1280 tubes gives (for $\omega$ in $\mathrm{cm}^{-1}$ and $D$ in $\AA$ ):

$$
\omega_{\mathrm{RB}}=2243 / D
$$

Some deviations from this law are noted for ultra narrow tubes. Two HE modes of chiral tubes have frequencies depending both on the chirality and diameter:

$$
\omega_{\mathrm{HE}}^{\mathcal{Z}}=1588+\frac{59.8 \cos 6 \theta}{D^{2}}, \quad \omega_{\mathrm{HE}}^{\mathcal{A}}=1588-\frac{757.6 \cos \theta}{D^{2}}-\frac{1069.9 \cos \theta}{D^{4}} .
$$

In achiral tubes there is a single totally symmetric high-energy mode (Table 2.3). Their frequencies are also given by (2.38): while $\omega_{\mathrm{HE}}^{\mathcal{Z}}$ gives the zig-zag HE mode for the chiral angle $\theta=0$, the armchair HE mode is obtained from $\omega_{\mathrm{HE}}^{\mathcal{A}}$ for $\theta=30^{\circ}$.

Usually it is assumed that the RB mode has only radial components, while the HE mode is circumferal in armchair tubes and longitudinal in zigzag tubes (thus, in chiral tubes one is considered as circumferal and the other one as longitudinal). However, symmetry in general forbids only circumferal components in zig-zag tubes and longitudinal ones in the armchair cases. This is confirmed by precise numerical calculations [31]: the RB small longitudinal component depends on the chiral angle $\theta$ and decreases with the tube diameter $D$. Indeed, in the armchair tubes, as the atoms are in the horizontal mirror planes, $\sigma_{\mathrm{h}}$ even (parity + ) and odd modes $(-)$ have vanishing longitudinal and horizontal components, respectively, i.e. vibrations are either perpendicular or parallel to the tube axis. Analogously, in zig-zag tubes the 


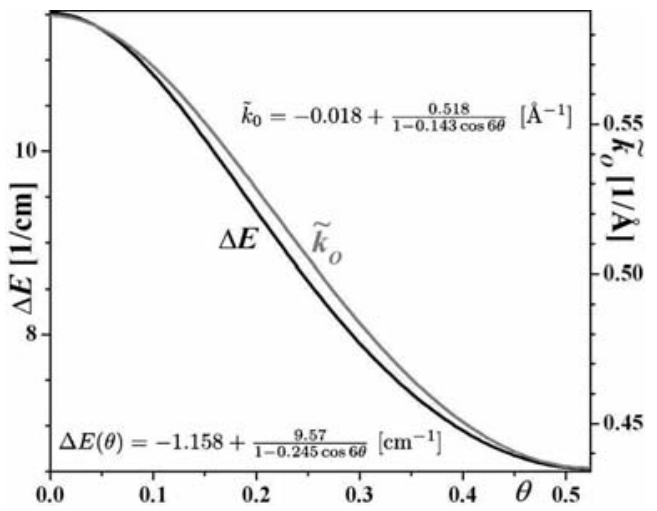

Fig. 2.14. Overbending $\Delta E$ and its maximum position $\tilde{k}_{0}$ as a function of the chiral angle $\theta$ for the 754 SWNTs (among 1280 tubes with diameters within 2.8$50.0 \AA$, those with maximal $D$ for given $\theta$ are selected). The data are precisely fitted by the given expressions

atoms are in the vertical mirror planes; thus the $\sigma_{\mathrm{v}}$-even $(A)$ displacements are in these planes, while the odd ones $(B)$ are circumferal. In addition to these symmetry constraints, HE mode(s) are perpendicular to the RB modes. Note that $\Pi_{\mathrm{h}}= \pm 1$ characterizes all $k=0$ modes, while $\Pi_{\mathrm{v}}= \pm 1$ is realized along the whole bands with $m=0, n$.

In contrast to almost all covalent solids, the graphite phonon branch corresponding to the longitudinal high energy optic mode at the $\Gamma$ point has a local minimum. This feature is usually referred to as "overbending", since the local maxima appear outside the $\Gamma$ point inside Brillouin zone. The analogous property of the SWNT phonon dispersions [31] is essential for the theoretical interpretation [64] of the dependence of the first-order Raman peak's position and double-peak structure of the high-energy mode $\left(\approx 1600 \mathrm{~cm}^{-1}\right)$ on the laser excitation energy. Namely, these features are attributed to the double-resonant process coming from the phonon modes out of the $\Gamma$ point. In the view of the generally quite weak dispersion around $k=0$, the excitation energy dependence is expected to be very sensitive to the vibrational spectra details. In particular, the accurate theoretical investigation may help the experimental sample characterization of the SWNTs. Indeed, from the Raman measurements the slope of the excitation energy dependence of the high-energy mode frequency can be obtained, while the quantum theory of the double resonant scattering relates this dependence to the overbending position $k_{0}$, its absolute value $\Delta E=E\left(k_{0}\right)-E(0)$ and slope, and the phonon eigenvectors (which can be easily calculated for any SWNT).

The overbending is analyzed (Fig. 2.14) for a large number of SWNTs of all chiralities and diameters up to $50 \AA$. The overbending maximum is found for the band assigned by $\tilde{m}=0$, at the helical wave vector $\tilde{k}_{0}$ depending on the tube chirality. Quantum numbers $k$ and $m$ are readily found by (2.23) for 
each SWNT, despite somewhat cumbersome general expression; e.g. only for the achiral tubes $m=0$. The absolute value $\Delta E$ is strongly chirality dependent, while for a fixed chiral angle, although it decreases with the diameter, it rapidly saturates for the very thin tubes, showing no further diameter dependence. Thus, the maximal overbending of $11.5 \mathrm{~cm}^{-1}$ is in the zig-zag tubes and the minimal $\left(6.50 \mathrm{~cm}^{-1}\right)$ in the armchair tubes.

\subsubsection{Commensurate Double-Wall Nanotubes}

\section{The Dynamical Matrix and Its Reduction}

Counting firstly the atoms of the wall $\mathrm{W}$, and after that the atoms of $\mathrm{W}^{\prime}$, the dynamical matrix obtains the block structure

$$
\mathcal{D}=\mathcal{D}_{\mathrm{WW}^{\prime}}^{0}+\mathcal{D}_{\mathrm{WW}^{\prime}}^{\prime}, \quad \mathcal{D}_{\mathrm{WW}}^{0}=\left(\begin{array}{cc}
\mathcal{D}_{\mathrm{W}}^{0} & 0 \\
0 & \mathcal{D}_{\mathrm{W}^{\prime}}^{0}
\end{array}\right), \quad \mathcal{D}_{\mathrm{WW}^{\prime}}^{\prime}=\left(\begin{array}{cc}
d_{\mathrm{WW}^{\prime}} & \mathcal{D}_{\mathrm{WW}^{\prime}} \\
\mathcal{D}_{\mathrm{W}^{\prime} \mathrm{W}} & d_{\mathrm{WW}^{\prime}}
\end{array}\right) .
$$

Here, $\mathcal{D}_{\mathrm{WW}}$ consists of the submatrices $\mathcal{D}_{\alpha \beta^{\prime}}$ comprising the intralayer coupling, as well as the transposed matrix $\mathcal{D}_{\mathrm{W}^{\prime} \mathrm{W}} ; \mathcal{D}_{\mathrm{W}}^{0}$ and $\mathcal{D}_{\mathrm{W}^{\prime}}^{0}$ are the dynamical matrices of the isolated walls. Finally, $d_{\mathrm{WW}^{\prime}}$ (and analogously $d_{\mathrm{W}^{\prime} \mathrm{W}}$ ) is the interlayer coupling dependent diagonal matrix; in the view of the translational sum rules for isolated and coupled walls, $\mathcal{D}_{\alpha \alpha}^{0}=-\sum_{\beta(\neq \alpha)} \mathcal{D}_{\alpha \beta}$ and $\mathcal{D}_{\alpha \alpha}=\mathcal{D}_{\alpha \alpha}^{0}-d_{\alpha \alpha}$, its elements are $d_{\alpha \alpha}=-\sum_{\beta^{\prime}} \mathcal{D}_{\alpha \beta^{\prime}}$.

Assuming the intralayer interaction unaffected, the same force constants model (Sect. 2.3.5) as for SWNTs is used. The interlayer submatrices are derived from the potential (2.14) in the Hessian form:

$$
\mathcal{D}_{\alpha \beta^{\prime}}=\frac{\partial^{2} v\left(\boldsymbol{r}_{\alpha}-\boldsymbol{r}_{\beta}^{\prime}\right)}{\partial x_{i}^{\alpha} \partial x_{j}^{\beta^{\prime}}}
$$

The dynamical matrix is invariant under the symmetries of DWCN, which is used to facilitate the diagonalization. If DWCN is incommensurate, then it has point group symmetry, and its finiteness prevents efficient reduction; therefore, only finite tubes, with at most a few hundred atoms, can be numerically studied. On the contrary, infinite line groups enable consideration of perfect infinite commensurate DWCNs. Therefore, the results presented obtained by the methods described in Sect. 2.3.1 refer to CDWNTs. Recall that the symcell of the DWNT contains many atoms, in contrast to SWNTs generated by a single atom. This is a consequence of greatly reduced rototranslational symmetry (in comparison to SWNTs), and therefore the range of $m$ usually consists only of the special values 0 and $Q / 2$; the corresponding states are even or odd in $z$-reversal parities. As discussed in Sect. 2.3.2, this is manifested as a large number of singularities in the density of states (Fig. 2.15). 


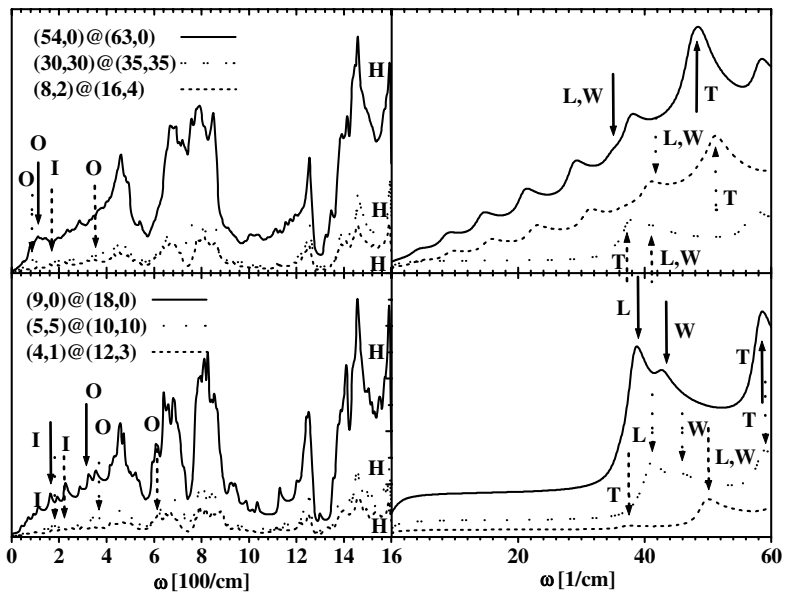

Fig. 2.15. Phonon density of states of CDWNTs with characteristic peaks L, T, W, I, O and H, denoting LRL, TRL, WRL, in- and out-of-phase BL and HE modes, respectively. Low frequency region is on the right

\section{Perturbative Interpretation}

One of the main common characteristics of CDWCN phonon bands is nicely illustrated by the example of $(5,5) @(10,10)$ in Fig. 2.16 ; it is notable that the branches of $\mathrm{W} @ \mathrm{~W}^{\prime}$ resemble the union of branches of $\mathrm{W}$ and $\mathrm{W}^{\prime}$, which would be obtained in the case of noninteracting walls. This is a consequence of the considerably lower interlayer interaction (2.14), in comparison to the intralayer force constants field. Accordingly, the elements of submatrices $\mathcal{D}_{\alpha \beta^{\prime}}$ are much less than those of $\mathcal{D}_{\alpha \beta}$ (unless $\alpha=\beta$ ), and may be considered as a perturbation to the dynamical matrix of the noninteracting walls. This invokes fruitful interpretation of CDWNT modes in terms of the modes of the isolated layers $[54,65]$.

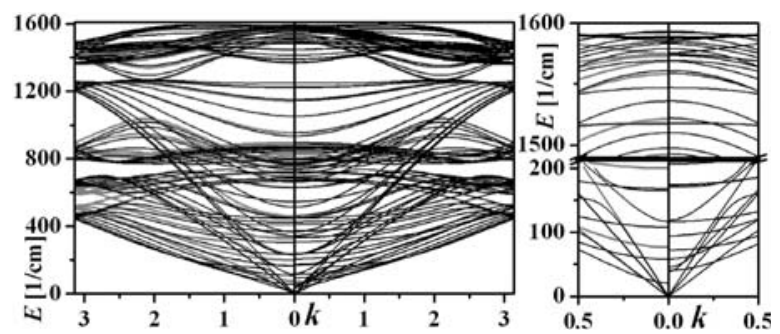

Fig. 2.16. Phonon bands of $(5,5) @(10,10)$ (right), $(5,5)$ (left, gray) and $(10,10)$ (left, black). The differences between the union of the phonon branches of $(5,5)$ and $(10,10)$ and the phonon branches of CDWCN $(5,5) @(10,10)$ decrease with frequency (from $10^{2} \mathrm{~cm}^{-1}$ for RL modes, to $10^{-3} \mathrm{~cm}^{-1}$ for the HE modes) 
Obviously, in view of (2.39) the interlayer interaction matrix $\mathcal{D}_{\mathrm{WW}^{\prime}}^{\prime}$ is a perturbation of the noninteracting wall dynamical matrix $\mathcal{D}_{\mathrm{WW}^{\prime}}^{0}$. In the basis $\left\{|a\rangle,\left|a^{\prime}\right\rangle\right\}$ ( $a$ and $a^{\prime}$ enumerate modes of $\mathrm{W}$ and $\mathrm{W}^{\prime}$ ) of the normal modes of noninteracting walls $\mathcal{D}_{\mathrm{WW}^{\prime}}^{0}$ is diagonal, with eigen energies $\omega_{a}^{2}$ and $\omega_{a^{\prime}}^{2}$, while the perturbation retains the same structure. However, the off-diagonal matrix elements $\kappa_{a a^{\prime}}=\sqrt{m}\left\langle a\left|\mathcal{D}_{\mathrm{WW}^{\prime}}^{\prime}\right| a^{\prime}\right\rangle \sqrt{m^{\prime}}$ take the role of the coupling of the two oscillatory modes $|a\rangle$ and $\left|a^{\prime}\right\rangle$. Recall that the normal mode basis is weighted by the square roots of the length mass densities (2.7) of the walls: $m=c D$ and $m^{\prime}=c D^{\prime}$. If modes $|a\rangle$ and $\left|a^{\prime}\right\rangle$ are not coupled to the others, the corresponding two-dimensional submatrix of $\mathcal{D}_{\mathrm{WW}^{\prime}}$ can be diagonalized independently of the rest of $\mathcal{D}_{\mathrm{WW}^{\prime}}$. This submatrix is the dynamical matrix [65] of the two oscillators with frequencies $\omega_{a}^{2}$ and $\omega_{a^{\prime}}^{2}$, and masses $m^{\prime}$ and $m$, coupled by the oscillator with force constant $\kappa_{a a^{\prime}}$ :

$$
\mathcal{D}_{a a^{\prime}}=\left(\begin{array}{cc}
\omega_{a}^{2}-\kappa_{a a^{\prime}} / m & \kappa_{a a^{\prime}} / \sqrt{m m^{\prime}} \\
\kappa_{a a^{\prime}} / \sqrt{m m^{\prime}} & \omega_{a^{\prime}}^{2}-\kappa_{a a^{\prime}} / m^{\prime}
\end{array}\right) .
$$

Hence, the modes of the two coupled isolated walls become two DWNT modes with frequencies

$$
\Omega_{ \pm}^{2}=\frac{1}{2}\left[\omega_{a}^{2}+\omega_{a^{\prime}}^{2}+\frac{\kappa_{a^{\prime} a}}{\mu_{+}} \pm \sqrt{\left(\omega_{a}^{2}-\omega_{a^{\prime}}^{2}\right)^{2}+2 \frac{\kappa_{a^{\prime} a}}{\mu_{-}}\left(\omega_{a}^{2}-\omega_{a^{\prime}}^{2}\right)+\frac{\kappa_{a^{\prime} a}^{2}}{\mu_{+}^{2}}}\right],
$$

where $\mu_{ \pm}=m^{\prime} m /\left(m \pm m^{\prime}\right)$. The higher frequency $\Omega_{+}$corresponds to the out-of-phase oscillations of the walls and $\Omega_{-}$to the in-phase ones. Note again that this simple approach is plausible only for the pairs of modes negligibly mixed with other modes, and such pairs may be singled out by symmetry. In fact, the interaction potential is invariant under all the symmetries of both walls, as obvious from (2.11), and further elucidated in Sect. 2.6. Therefore, the modes with the same quantum numbers are coupled much more strongly than differently assigned modes. Accordingly, the perturbative interpretation is plausible for the pairs of equally assigned modes, with frequencies well separated from the other modes with the same quantum numbers. Further, as the interaction field is effectively averaged in the rapid oscillations and thus partly cancelled, the coupling of low frequency modes is in general more intensive.

\section{Rigid Layer Modes}

The acoustic modes are equally assigned in all achiral SWNTs by mutually different quantum numbers. Since their zero frequency is much less than the other modes of same assignation, the pairs of LA, TA and TW modes are suitable for perturbative analysis. Hence, substituting $\omega_{a}=\omega_{a^{\prime}}=0$ in $(2.42)$ one finds 


$$
\Omega_{-}=0, \quad \Omega_{+}(D)=\sqrt{\frac{2 \kappa}{c} \frac{D+\Delta / 2}{D(D+\Delta)}},
$$

i.e. one acoustic and one low frequency optic DWNT mode for each SWNT acoustic pair. In both modes the layers vibrate as rigid bodies, in-phase in the acoustic and out-of-phase in the optic mode. Therefore, the out-of-phase vibrations are rigid-layer modes (Fig. 2.15), transversal (TRL), longitudinal (LRL) and twisting (TWRL), in correspondence to their in-phase TA, LA and TW counterparts.

The branches starting at $k=0$ with acoustic modes are twofold, except that the TA branch is fourfold for $\mathcal{Z} \mathcal{Z}$ and $\mathcal{A} \mathcal{A}$ CDWNTs (other tubes have two close TA branches). These bands are linear in $k$ near $k=0$, with the slope almost independent of the tube (the differences are less than 1\%) and close to that for SWNTs, giving the sound velocities $v_{\mathrm{TA}}=9.54 \mathrm{~km} \mathrm{~s}^{-1}$, $v_{\mathrm{LA}}=20.64 \mathrm{~km} \mathrm{~s}^{-1}$ and $v_{\mathrm{TW}}=15.18 \mathrm{~km} \mathrm{~s}^{-1}$.

The coupling constant $\kappa$ is itself a function of the tube diameter and the interlayer distance. The number of interacting atoms per unit length increases with $D$, yielding an increase in $\kappa$ as well. Proposing a polynomial function $\kappa(D)$, it turns out that for LRL and TWRL modes the first order polynomial fits the numerical data well [66], while for TRL modes a quadratic term must be added. Thus, with the convenient form $\kappa(D)=c \omega_{\infty}^{2}\left(\delta_{0}+D+\gamma D^{2}\right) / 2$, the rigid layer frequencies are:

$$
\Omega_{+}(D)=\omega_{\infty} \sqrt{\frac{\left(D+\delta_{0}+\gamma D^{2}\right)(D+\Delta / 2)}{D(D+\Delta)}} .
$$

The model is clearly justified by Fig. 2.17 comparing the numerical results to the analytic form (2.44) with the parameters given in Table 2.4 (assuming

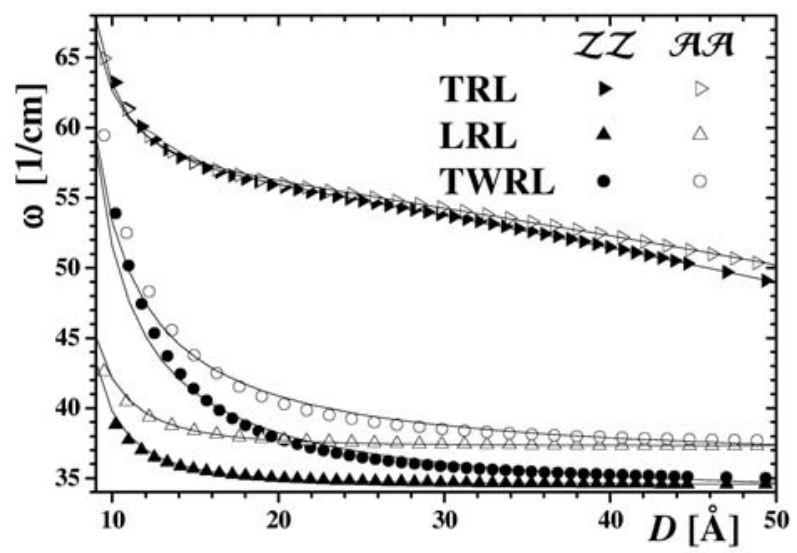

Fig. 2.17. Rigid layer mode frequencies vs. DWNT diameter. The solid lines are fitted curves 
Table 2.4. Fitting parameters for RL modes (see (2.44) and Fig. 2.17).

\begin{tabular}{|c|c|c|}
\hline & $(n, 0) @(n+9,0)$, & $(n, n) @(n+5, n$ \\
\hline & $\begin{array}{lll}\omega_{\infty} & \delta_{0} & \gamma \\
\end{array}$ & $\omega_{\infty} \quad \delta_{0} \quad \gamma$ \\
\hline LRL & $34.7-4.00 \quad 0$ & $37.4-4.00 \quad 0$ \\
\hline TWRL & $\begin{array}{lll}33.8 & 1.33 & 0\end{array}$ & $\begin{array}{lll}36.0 & 1.33 & 0\end{array}$ \\
\hline TRL & $62.5-4.67-0.0068$ & $61.7-4.39-0.006$ \\
\hline
\end{tabular}

frequency in $\mathrm{cm}^{-1}$ and the interior-wall diameter in $\AA$ ). Additional verification may be found in the large $D$ limit, when the graphene values should be reproduced. Then LRL and TWRL modes become two degenerate $A$ point $\left(k_{z}=\pi / c, k_{x}=k_{y}=0\right)$ modes of graphite [67], describing rigid out-of-phase vibrations (in two perpendicular directions) of the adjacent graphite layers. Indeed, as $\gamma=0$, for both modes and for both types of achiral DWNTs $\Omega_{+}(\infty)=\omega_{\infty}=35 \mathrm{~cm}^{-1}$, matching the singled out graphite frequency. Note that this matching is dominantly due to symmetry. As discussed in detail in Sect. 2.6, symmetry completely determines the patterns of wall-wall interaction, particularly the periodicity and this way its slopes in $\Phi$ and $Z$ around stable configuration. This yields correct experimental frequencies for graphite as the large $D$ DWNT limit, despite the fact that the Lenard-Jones potential used is assumed to be too smooth to describe directly out-of-phase rigid-layer modes of the adjacent graphite layers.

These results may be of experimental interest, since TRL and LRL modes are IR active (TWRL is also IR active for tubes with at least one chiral wall), and correspond to the density of state singularities (Fig. 2.15).

The rigid layer modes influence the low temperature heat capacity. In fact, if there were no interlayer interaction, the DWNT heat capacity would be equal to that of the walls, as an average of almost the same quantities. However, the coupling of the walls' acoustic modes (altogether eight modes) produces only four DWNT acoustic modes, while another four become optical. Therefore, the heat capacity is close to that of the layers, except in the low temperature region, contributed by the acoustic branches only, where it is significantly lowered (Fig. 2.18). This agrees nicely with the available experimental data $[58,68]$.

\section{Breathing-Like and High-Energy Modes}

The same arguments as for the acoustic modes of the layers justify perturbative interpretation for the totally symmetric $(k=m=0$, even in all parities) radial breathing and high-energy modes (Fig. 2.19), being mutually well separated by frequency. The frequencies of the resulting [65] in-phase and out-ofphase breathing-like (BL) modes are found by substituting frequencies (2.37) in (2.42). The numerical results [66], well fitted by $\kappa_{\mathrm{BL}}=-246404+38799 D$, are in accordance with earlier predictions [54] and match the experimen- 


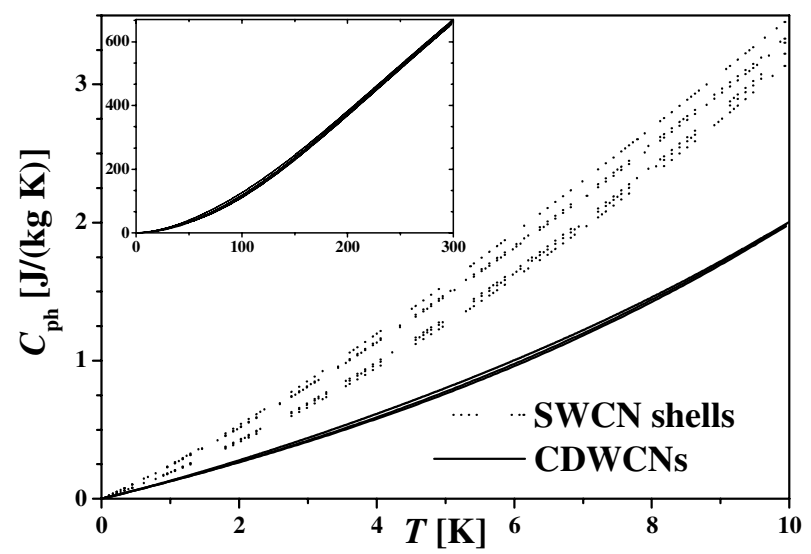

Fig. 2.18. Heat capacity of double wall tubes $\mathcal{A A}_{5}, \mathcal{Z} \mathcal{Z}_{9}(8,2) @(16,4)$ and their SWNT constituents in the low temperature regime, and up to $300 \mathrm{~K}$ (inset)

tal results [69-71]. As for the high energy branches, it is known from measurements that they hardly differ from those of SWNT. Also, the difference not greater than $10^{-3} \mathrm{~cm}^{-1}$ is found numerically. Fit by (2.42) with isolated wall frequencies (2.38) gives coupling constants $\kappa_{\mathrm{HE}}^{\mathcal{Z Z}}=-25875+6528 D$ and $\kappa_{\mathrm{HE}}^{\mathcal{A \mathcal { A }}}=9329+6307 D$.

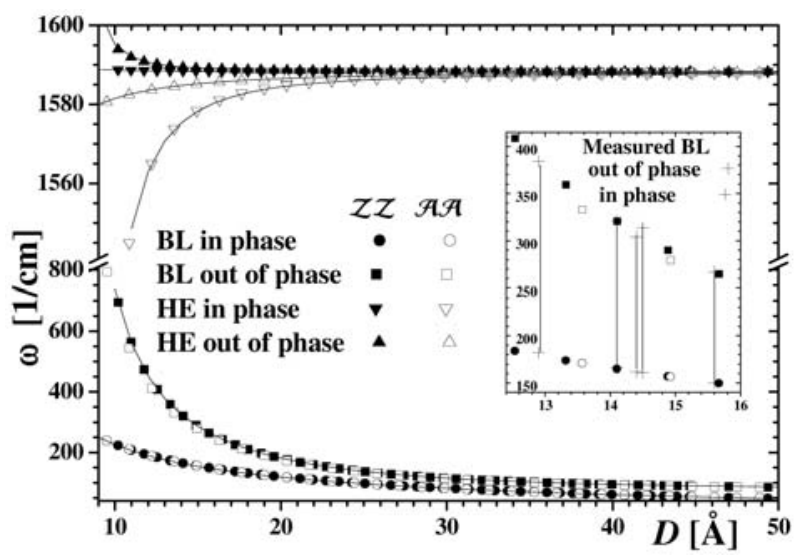

Fig. 2.19. Rigid layer (left) and breathing-like and high-energy (right) frequencies vs. DWNT diameter. The solid lines are fitted curves. Inset: comparison of the calculated and the experimental results [71] 


\subsection{Symmetry Breaks Friction: Super-Slippery Walls}

The symmetry of double-wall nanotubes gives a profound insight to the interlayer interaction, as well as an efficient way to estimate it. Such analysis clarifies the main characteristics of this potential: while in general it is quite weak, a variety of the potential shapes may be created by the appropriate choice of the chirality and length of the walls. Therefore, DWNTs are perfect bearings, and attractive candidates for nano-electromechanical devices [14, $72]$.

\subsubsection{Symmetry and Interaction}

To enlighten symmetry properties of the interwall interaction potential $V(\Phi, Z)$, at first Eq. (2.11) is rewritten as the sum

$$
V(\Phi, Z)=\sum_{t^{\prime} s^{\prime} u^{\prime}} V_{\mathrm{in}}\left(\boldsymbol{r}_{t^{\prime} s^{\prime} u^{\prime}}^{\prime}\right)
$$

of the energy of the outer wall atoms in the potential created by the inner wall:

$$
V_{\mathrm{in}}(\boldsymbol{r})=\sum_{t s u} v\left(\boldsymbol{r}_{t s u}, \boldsymbol{r}\right)
$$

This form manifests that $V_{\text {in }}$ is invariant under the inner tube symmetry group. Therefore it can be expanded over the basis of invariant functions:

$$
V_{\mathrm{in}}(\boldsymbol{r})=\sum_{M \geq 0, \omega} \alpha_{\omega}^{M}\left(D^{\prime}\right) C_{\omega}^{M}(\varphi, z)
$$

Here, the basis consists of the functions $C_{\omega}^{M}(\varphi, z)=\cos (M \varphi+2 \pi \omega z)$; rotational and screw axis invariance, respectively, restricts [73] nonnegative integers $M$ and real numbers $\omega$ by:

$$
M=0 \quad(\bmod n), \quad M r+\omega n a=0 \quad(\bmod q) .
$$

Due to the summation in $(2.45)$, the total potential $V(\Phi, Z)$ is also invariant under the outer wall symmetries, and therefore under all the transformations generated by the symmetries of both walls. Consequently, to $V(\Phi, Z)$ contribute only the terms of (2.47) subdued to the additional restrictions of the form (2.48), but with the outer wall parameters $q^{\prime}, r^{\prime}, n^{\prime}$ and $a^{\prime}$. Taking this into account, the summation in (2.45) is easily performed; the total potential per atom of an infinitely long DWNT is:

$$
v_{\infty}(\Phi, Z)=2 \sum_{M \geq 0, \omega}^{\prime \prime} \alpha_{|\omega|}^{M} \cos (M \Phi+2 \pi \omega Z) \cos \left(M \varphi_{000}+2 \pi \omega z_{000}\right) .
$$


The double prime indicates two pairs of restrictions (2.48), imposed by the roto-translational symmetries of the walls on $M$ and $\omega$.

Mutually commuting roto-translational transformations of the walls together generate the breaking group $\boldsymbol{L}_{\mathrm{B}}^{\mathrm{R}}[13]$. Note that if walls did not interact, the system would be invariant under any pair of independently performed symmetries of the walls, i.e. under the product group $\boldsymbol{L}_{\mathrm{W}}^{\mathrm{R}} \times \boldsymbol{L}_{\mathrm{W}^{\prime}}^{\mathrm{R}}$. Hence, the interaction itself imposes the symmetry breaking from the product to the intersection (2.12). Denoting by $|\boldsymbol{L}|$ the number of transformations in $\boldsymbol{L}$, the symmetries of the noninteracting and interacting walls are related by:

$$
\left|\boldsymbol{L}_{\mathrm{W} W^{\prime}}^{\mathrm{R}}\right|\left|\boldsymbol{L}_{\mathrm{B}}^{\mathrm{R}}\right|=\left|\boldsymbol{L}_{\mathrm{W}}^{\mathrm{R}}\right|\left|\boldsymbol{L}_{\mathrm{W}^{\prime}}^{\mathrm{R}}\right|
$$

Thus, while the roto-translational symmetry of the DWNT is highly reduced in comparison to that of the walls, the breaking group, being the symmetry group of the interaction potential (2.49), is much greater. Particularly, the periods $A_{\mathrm{B}}$ and $2 \pi / N_{\mathrm{B}}$ (along $Z$ and $\Phi$, respectively) of the interaction potential decrease with the periods $A$ and $2 \pi / N$ of DWNT. For the commensurate DWNTs, the breaking group is the line group [13]:

$$
\boldsymbol{L}_{\mathrm{B}}^{\mathrm{R}}=\boldsymbol{T}_{Q_{\mathrm{B}}}^{R_{\mathrm{B}}}\left(A_{\mathrm{B}}\right) \boldsymbol{C}_{N_{\mathrm{B}}},
$$

with the parameters (compare to (2.13) for $Q, N$ and $A)$ :

$$
\begin{aligned}
& N_{\mathrm{B}}=\frac{n n^{\prime}}{N} \frac{\sqrt{\tilde{q} \tilde{q}^{\prime}}}{\operatorname{GCD}\left(\frac{r^{\prime} \hat{a} n-r \hat{a}^{\prime} n^{\prime}}{N}, \sqrt{\tilde{q} \tilde{q}^{\prime}}\right)}, \quad Q_{\mathrm{B}}=\frac{q q^{\prime}}{\operatorname{GCD}\left(q, q^{\prime}\right)}, \quad A_{\mathrm{B}}=\frac{a a^{\prime}}{A N} \frac{Q_{\mathrm{B}} Q}{q q^{\prime}}, \\
& \frac{R_{\mathrm{B}}}{\operatorname{GCD}\left(q, q^{\prime}\right)}=\frac{\left(r^{\prime} \hat{a} q-r \hat{a}^{\prime} q^{\prime}\right) \hat{a}^{\prime \phi(\hat{a})}+r q^{\prime} \hat{a^{\prime}}}{\hat{a} \hat{a}^{\prime}} \quad\left(\bmod \operatorname{GCD}\left(N \tilde{q} \tilde{q}^{\prime}, r^{\prime} \hat{a} q-r \hat{a}^{\prime} q^{\prime}\right)\right) .
\end{aligned}
$$

By convention, $R_{\mathrm{B}}$ is the (unique) solution of the last equation, which is coprime with $Q_{\mathrm{B}}$ and less than $Q_{\mathrm{B}}$. In the incommensurate case the breaking group is:

$$
\boldsymbol{L}_{\mathrm{B}}^{\mathrm{R}}=\boldsymbol{C}_{Q_{\mathrm{B}}} \boldsymbol{T}(0)
$$

Here, $\boldsymbol{T}(0)$ is the translational group generated by the incommensurate periods $a$ and $a^{\prime}$, i.e. including the translations for $t a+t^{\prime} a^{\prime}$ for all the integers $t$ and $t^{\prime}$. This is the quasi-continual group, as for any real $x$ and arbitrary small $\varepsilon$, there are $t$ and $t^{\prime}$ such that $\left|t a+t^{\prime} a^{\prime}-x\right| \leq \varepsilon$. Therefore, the invariance of $V(\Phi, Z)$ under the group $\boldsymbol{T}(0)$, in view with its physically indispensable continuity, implies that it is constant along $Z$. In fact, incommensurate tubes can be viewed as the limiting case: $A=\infty$, thus $A_{\mathrm{B}}=0$, while due to the $Z$ independence, the (fractional) translations do not affect the potential and $Q_{\mathrm{B}}$ takes the role of $N_{\mathrm{B}}$.

It is clear from the definition of the breaking group that two pairs (2.48) (one for each wall) of conditions on $M$ and $\omega$ are equivalent to the single such pair with the breaking group parameters $Q_{\mathrm{B}}, R_{\mathrm{B}}, N_{\mathrm{B}}$ and $A_{\mathrm{B}}$. As the breaking group is quite large with respect to the single-wall symmetry, this implies 
that most of the terms in (2.46) do not contribute to the interaction $V(\Phi, Z)$, meaning that the inter-shell friction is in general quite low. In addition, the rotational and translational breaking independently give rough insight to the interaction potential: large $N_{\mathrm{B}}$ leaves only high $M$ terms in (2.49), as well as small $A_{\mathrm{B}}$ allows only large $\omega$ terms. Together with the natural assumption that the amplitudes $\alpha_{|\omega|}^{M}$ rapidly decrease with $M$ and $\omega$ (being justified numerically [73]), this means that large rotational and translational breaking are manifested as slow varying potential along $\Phi$ and $Z$, respectively. Numerical calculations $[14,15,74,75]$ and recent experiments $[76,77]$ confirm this theoretical prediction; particularly, the static friction has been estimated [77] to be lower than $6 \cdot 10^{-15} \mathrm{~N} / \AA^{2}$.

As remarked in Sect. 2.2.2, most DWNTs are incommensurate (even $99.5 \%$ of the ones studied), with $Z$ independent interaction potential. Also in commensurate cases breaking is large, and despite several highly symmetric CDWNTs, potential is almost constant along one of the coordinates. Therefore, for nanomechanical applications it is interesting to consider DWNTs with one finite layer, providing additional variety of potential patterns. The summation over the atoms in (2.11) is slightly modified to include only $m$ outer wall monomers (the outer wall length is $\left.n^{\prime} a^{\prime}(m-1) / q^{\prime}\right)$ : $t^{\prime}=0,1, \ldots, m-1$, while all possible values of $u^{\prime}$ and $s^{\prime}$ are allowed as before. After performing this summation, the total interaction per atom is:

$$
\begin{aligned}
v_{m}(\Phi, Z)= & 2 \sum_{M \geq 0, \omega}^{\prime \prime} \alpha_{\omega}^{M} \cos (M \Phi+2 \pi \omega Z) \\
& \times \cos \left(M \varphi_{0}+2 \pi \omega z_{0}\right) \frac{\sin \left(\pi m \frac{r^{\prime} M+n^{\prime} a^{\prime} \omega}{q^{\prime}}\right)}{m \sin \left(\pi \frac{r^{\prime} M+n^{\prime} a^{\prime} \omega}{q^{\prime}}\right)} .
\end{aligned}
$$

Now the double prime restricts the summation to the solutions of the system:

$$
M=0 \quad(\bmod n) ; \quad M=0 \quad\left(\bmod n^{\prime}\right) ; \quad M r+\omega n a=0 \quad(\bmod q) .
$$

The horizontal $U$ axis of the receiver wall is in the middle of the ring with $\Phi_{m}=\Phi+\frac{2 \pi r^{\prime}}{q^{\prime}} \frac{m-1}{2}$ and $Z_{m}=Z+\frac{n^{\prime} a^{\prime}}{q^{\prime}} \frac{m-1}{2}$ (the infinite tube $U$ axis at $(\Phi, Z)$ is no longer a symmetry, as the monomers are above it only). In comparison to the infinite case, there is no restriction imposed on $M$ and $\omega$ by the other wall screw-axis. Consequently, the potential $v_{m}(\Phi, Z)$ can be viewed as $v_{\infty}(\Phi, Z)$ superposed to $v_{m}^{\text {fin }}(\Phi, Z)$ comprising the damped (factor $1 / m$ ) oscillations. Also, a resonant effect is observed from (2.53): whenever $m$ is a multiple of $q^{\prime} A / n^{\prime} a^{\prime}$ the dumping term vanishes.

\subsubsection{Numerical Results}

Several pairwise potentials are used in the literature for calculating the interlayer interaction $[14,15,74,75]$. This has caused quantitative differences of the results: e.g. for the energy barrier of the relative sliding of the 
walls in $(5,5) @(10,10)$ Charlier and Michenaud got $0.52 \mathrm{meV} /$ atom (longitudinal) and $0.23 \mathrm{meV} /$ atom (circumferal), and Palser $0.295 \mathrm{meV} /$ atom and $0.085 \mathrm{meV} /$ atom. Nevertheless, there is agreement that the relative sliding of two nested tubes is easy.

Here we discuss potential barriers calculated with the pairwise potential of the Van der Waals type (2.14). Except for a few highly symmetric achiral DWNTs, qualitatively the same results are obtained with another potential $[15,73]$ taking care of $\pi$-bonding. The amplitudes $\alpha_{\omega}^{M}$ are derived by fast Fourier transform of the potential $V_{\text {in }}$. The interior tube is, long at least $100 \AA$ (at least 40 elementary cells); the potential is scanned on the grid of $41 \times 41$ points for $\varphi$ and $z$ at the radius of the outer-wall. The constant term $\alpha_{0}^{0}$ is set to zero while the numerical error of the calculated interaction potential is estimated to be $10^{-10} \mathrm{meV}$.

The patterns shown in Fig. 2.20 illustrate the variety of potential shapes of DWNTs with infinite walls. There are basically two different patterns of the interaction potential. Firstly, "horizontal stripes" appear in all the studied commensurate DWNT with achiral walls, with the exception of $(5,5) @(10,10)$; in fact, due to the high incompatibility of the rotational symmetries of the layers with larger diameters, the calculated potential is almost $\Phi$ independent. Secondly, "vertical stripes" enabling super-slippery sliding along $Z$ are typical for the incommensurate tubes. A large number of commensurate tubes interact negligibly within the numerical precision, due to the symmetries of the highly incompatible layers, which is manifested as a perfectly flat potential surface.

Additional potential shapes appear in DWNTs with a finite outer wall (Fig. 2.21). Note that, depending on the chirality of the layers, the easiest sliding is allowed along helices with angles varying from $-90^{\circ}$ to $90^{\circ}$. Also, there are sharply defined minima or maxima. From (2.53) it is clear that a change in the length of the outer wall may result in a different pattern, due to $m$-dependance of $v_{m}^{\mathrm{fin}}(\Phi, Z)$. Also, the larger barrier reported [14] for the tubes $(5,5) @(15,4)$ and $(9,0) @(15,4)$ can be easily explained: the tube
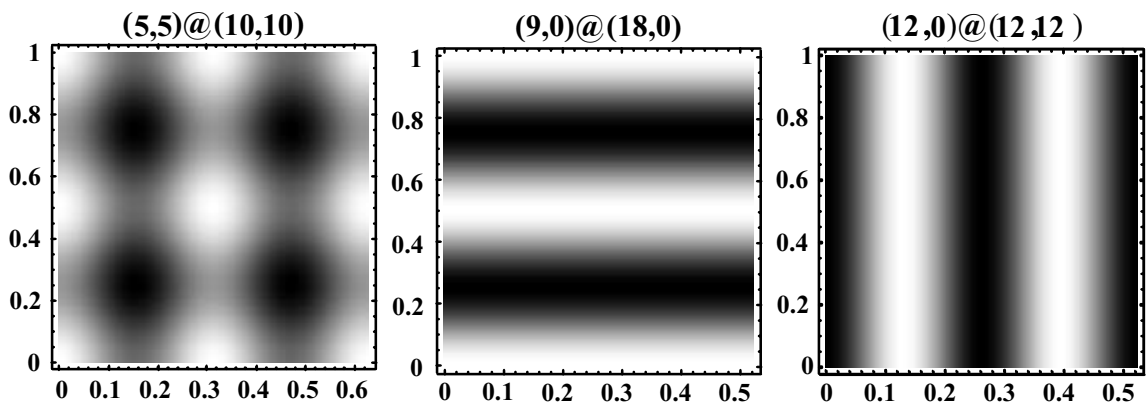

Fig. 2.20. Density plot of $V(\Phi, Z)$ for infinite $(5,5) @(10,10),(9,0) @(18,0)$ and $(12,0) @(12,12)(\Phi$ and $Z / a$ are along the vertical horizontal and vertical axes) 

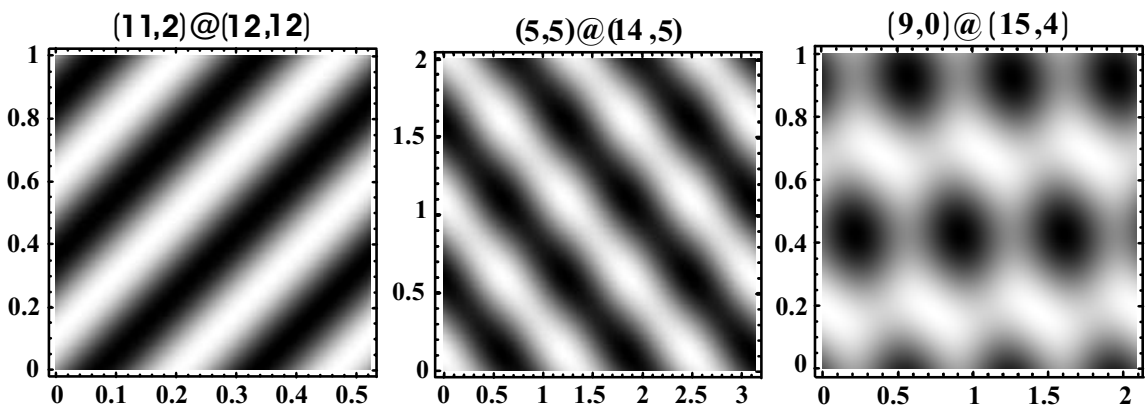

Fig. 2.21. Density plot of $V(\Phi, Z)$ for $(11,2) @(12,12), \quad(5,5) @(14,5)$ and $(9.0) @(14,5)$, with the outer walls of 40,194 and 301 monomers, respectively

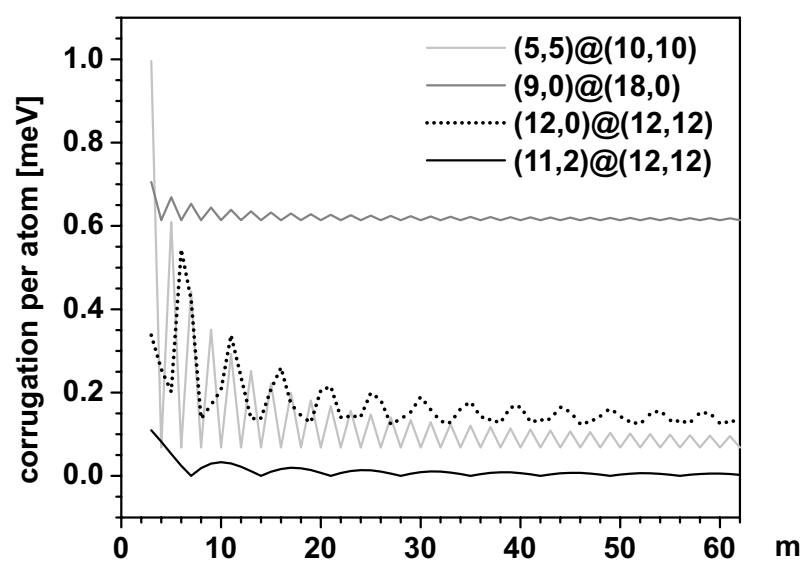

Fig. 2.22. Corrugation per atom as a function of $m$ for several DWNTs with the outer wall made of $m$ monomers

$(15,4)$, without rotational symmetry, does not restrict the values of $M$, and consequently $v_{m}^{\text {fin }}(\Phi, Z)$ contains all the $\omega \neq 0$ terms, including those with significant amplitudes $\alpha_{\omega}^{M}$.

To get a better insight into the possible "low energy cost motions" the corrugation for several tubes is calculated (Fig. 2.22). The resonant effect is clearly visible. For the $(11,2) @(12,12)$ tube $q^{\prime} A / n^{\prime} a^{\prime}=14$, and whenever $m$ is a multiple of 14 the corrugation per atom drops to its limiting $m=\infty$ value. For achiral commensurate DWNTs this effect is more pronounced, as it occurs for each even $m$. 


\section{References}

1. S. Iijima: Nature, 354, 56 (1991)

2. A. Thess, R. Lee, P. Nikolaev, H. Dai, P.Petit, J. Robert, C.H. Xu, Y.H. Lee, S.G. Kim, A.G. Rinzler, D.T. Colbert, G.E. Scuseria, D. Tomanek, J.E. Fischer and R.E. Smally: Science 273, 483 (1996)

C. Journet, W.K. Maser, P. Bernier and A. Loiseau: Nature 388, 756 (1997)

3. M.S. Dresselhaus, G. Dresselhaus and R. Saito: Phys. Rev. B 45, 6234 (1992)

4. N. Hamada, S. Sawada and A. Oshiyama: Phys. Rev. Lett. 68, 1579 (1992)

5. C.T. White, D.H. Robertson and J.W. Mintmire: Phys. Rev. B 47, 5485 (1993) J.W. Mintmire and C.T. White, Phys. Rev. Lett. 81, 2506 (1998)

6. R.A. Jishi, L. Venkataraman, M.S. Dresselhaus and G. Dresselhaus: Phys. Rev. B 16, 11176 (1995)

7. M. Damnjanović, I. Milošević, T. Vuković and R. Sredanović: Phys. Rev. B 60, 2728-2739 (1999)

8. M. Vujičić, I. Božović and F. Herbut: J. Phys. A 10, 1271 (1997)

9. I. Milošević and M. Damnjanović: Phys. Rev. B 47, 7805 (1993)

10. H. Abud and G. Sartori: Ann. Phys. 150, 307 (1983)

11. I. Cabria, W. Mintmire and C.T. White: Phys. Rev. B 67, 121406(R) (2003)

12. Y.E. Lozovik, A.M. Popov and A.V. Belikov: Phys. Sol. State 45, 1396 (2003)

13. M. Damnjanović, I. Milošević, E. Dobardžić, T. Vuković and B. Nikolić: J. Phys. A 36, 10349-10360 (2003)

14. R. Saito, R. Matsuo, T. Kimura, G. Dresselhaus G and M.S. Dresselhaus: Chem. Phys. Lett. 348, 187 (2001)

15. A. Kolmogorov, V. Crespi: Phys. Rev. Lett. 85, 4727 (2000)

16. E.P. Wigner: Group Theory and its Applications to the Quantum Mechanics of Atomic Spectra (Academic Press, New York 1959)

17. M. Damnjanović and I. Milošević: J. Phys. A 28, 1669-79 (1995)

18. M. Damnjanović, T. Vuković and I. Milošević: J. Phys A 33, 6561-72 (2000)

19. I. Milošević, A. Damjanović, and M. Damnjanović: Ch. XIV. In: Quantum Mechanical Simulation Methods in Studying Biological Systems, ed by D. Bicout and M. Field (Springer-Verlag, Berlin, Heidelberg, New York 1996)

20. M. Damnjanović, I. Milošević, T. Vuković and J. Maultzsch: J. Phys. A 36, 5707-17 (2003)

21. T. Vuković, I. Milošević and M. Damnjanović: Phys. Rev. B 65, 045418 (2002)

22. C. Kane and E.J. Mele: Phys. Rev. Lett. 78, 1932 (1997)

23. M. Damnjanović, T. Vuković and I. Milošević: Solid State Comm. 116, 265 (2000)

24. S. Tasaki, K. Maekawa and T. Yamabe: Phys. Rev. B 57, 9301 (1998)

25. S. Reich, J. Maultzsch and C. Thomsen: Phys. Rev. B 66, 035412 (2002)

26. M. Machón, S. Reich, C. Thomsen, D. Sánchez-Portal, P. Ordejón: Phys. Rev. B 66, 155410 (2002)

27. H.J. Liu and C.T. Chan: Phys. Rev. B 66, 115416 (2002)

28. D. Porezag, Th. Frauenheim, Th. Köhler, G. Seifert and R. Kaschner: Phys. Rev. B 51, 12947 (1995)

29. E. Schröder and P. Hyldgaard: Surface Sci. 532-5, 880 (2003)

30. O. Dubay and G. Kresse: Phys. Rev. B 67, 035401 (2003)

31. E. Dobardžić, I. Milošević, B. Nikolić, T. Vuković and M. Damnjanović: Phys. Rev. B 68, 045408 (2003) 
32. R.A. Jishi, L. Venkataraman, M.S. Dresselhaus and G. Dresselhaus: Chem. Phys. Lett. 209, 77 (1993)

33. M. Born and K. Huang: Dynamical Theory of Crystal Lattices (Oxford University Press, Oxford, 1954)

34. O. Madelung: Solid State Theory, (Springer-Verlag, Berlin, Heidelberg, New York 1978)

35. R. Saito, T. Takeya, T. Kimura, G. Dresselhaus and M.S. Dresselhaus: Phys. Rev. B 57, 4145 (1998)

36. O. Jost, A.A. Gorbunov, W. Pompe, T. Pichler, R. Friedlein, M. Knupfer, M. Reibold, H.-D. Bauer, L. Dunsch, M.S. Golden and J. Fink: Appl. Phys. Lett. 75, 2217 (1999);

S. Kazaoui, N. Minami, R. Jacquemin, H. Kataura and Y. Achiba: Phys. Rev. B 60, 13339 (1999);

X. Liu, T. Pichler, M. Knupfer, M.S. Golden, J. Fink, H. Kataura and Y. Achiba: Phys. Rev. B 66, 045411 (2002)

37. H. Kuzmany, W. Plank, M. Hulman, Ch. Kramberger, A. Grüneis, Th. Pichler, H. Peterlik, H. Kataura and Y. Achiba: Eur. Phys. J. B 22, 307 (2001)

38. H. Ajiki and T. Ando: Physica B 201, 349 (1994)

39. W.A. de Heer, W.S. Bacsa, A. Chatelain, T. Gerfin, R. Humphrey-Baker, L. Forro and D. Ugarte: Science 268, 845 (1995)

40. F. Bommeli, L. Degioergi, P. Wachter, W.S. Bacsa, W.A. de Heer and L. Forro: Solid State Commun. 99, 513 (1996)

41. H.H. Gommans, J.W. Alldredge, H. Tashiro, J. Park, J. Magnuson and A.G. Rinzler: J. Appl. Phys. 88, 2509 (2000);

J. Hwang, H.H. Gommans, A. Ugawa, H. Tashiro, R. Haggenmueller, K.I. Winey, J.E. Fischer, D.B. Tanner and A.G. Rinzler: Phys. Rev. B 62, R13310 (2000);

S. Reich, C. Thomsen, G.S. Duesberg and S.Roth: Phys. Rev. B 63, 041401(R) (2001)

42. M.F. Lin: Phys. Rev. B 62, 13153 (2000)

43. I. Božović, N. Božović and M. Damnjanović: Phys. Rev. B 62, 6971 (2000)

44. I. Milošević, T. Vuković, S. Dmitrović and M. Damnjanović: Phys. Rev. B 67, 165418 (2003)

45. S. Sawada and N. Hamada: Solid State Commun. 83, 917 (1992)

46. Z.K. Tang, H.D. Sun, J. Wang, J. Chen and G. Li: Appl. Phys. Lett. 73, 2287 (1998)

N. Wang, Z.K. Tang, G.D. Li and J.S. Chen: Nature (London) 408, 50 (2000)

47. Z.M. Li, Z.K. Tang, H.J. Liu, N. Wang, C.T. Chan, R. Saito, S. Okada, G.D. Li, J.S. Chen, N. Nagasawa and S. Tsuda: Phys. Rev. Lett. 87, 127401 (2001)

48. Y.F. Chan, H.Y. Peng, Z.K. Tang and N. Wang: Chem. Phys. Lett. 369, 541 (2003)

49. X.P. Yang, H.M. Weng and J. Dong: Eur. Phys. J. B 32, 345 (2003)

50. I. Milošević, B. Nikolić and M. Damnjanović: Phys. Rev. B 69, 113408 (2004)

51. S.M. Bachilo, M.S. Strano, C. Kittrell, R.H. Hauge, R.E. Smalley, R.B. Weisman: Science 2982361 (2002)

C.L. Kane and E.J. Mele: Phys. Rev. Lett. 90207401 (2003)

C.D. Spataru, S. Ismail-Beigi, L.X. Benedict and S.G. Louie: condmat/0310220v1 
52. L.X. Benedict, S.G. Louie and M.I. Cohen: Phys. Rev. B 52, 8541 (1995);

G.S. Duesberg, I. Loa, M. Burghard, K. Syassen, S. Roth: Phys. Rev. Lett. 85, $5436(2000)$;

A. Jorio, A.G. Souza Filho, V.W. Brar, A.K. Swann, M.S. Ünlü, B.B. Goldberg, A. Right, J.H. Hafner, C.M. Lieber, R. Saito, G. Dresselhaus, M.S. Dresselhaus: Phys. Rev. B 65, 121402(R) (2002)

53. O.E. Alon: Phys. Rev. B. 63, 201403(R) (2001)

54. V.N. Popov and L. Henrard: Phys. Rev. B 65, 235415 (2002)

55. G.D. Mahan: Phys. Rev. B. 65, 235402 (2002)

56. R. Saito, G. Dresselhaus, M. Dresselhaus: Physical Properties of Carbon Nanotubes (Imp. College Press, London 1998)

57. L.X. Benedict, S.G. Louie and M.L. Kohen: Solid State Commun. 100, 177 (1996)

58. A. Mizel, L.X. Benedict, L.M. Cohen, S.G. Louie, A. Zettl, N.K. Budraa and W.P. Beyermann: Phys. Rev. B 60, 3264 (1999)

59. A. Charlier, E. McRae, M.-F. Charlier, A. Spire and S. Forster: Phys. Rev. B $\mathbf{5 7}, 6689$ (1998)

60. J. Hone, B. Batlogg, Z. Benes, A.T. Johnson, J.E. Fischer: Sciense 289, 1730 (2000)

61. J.C. Lasjaunias, K. Biljaković, Z. Benes, J.E. Fischer and P. Monceau: Phys. Rev. B 65, 113409 (2002)

62. J.L. Birman: Theory of Crystal Space Groups and Infra-Red and Raman Lattice Processes of Insulating Crystals, (Springer-Verlag, Berlin, Heidelberg, New York 1974)

63. D.A. Long: Raman Spectroscopy (McGRaw-Hill, New York 1977)

64. C. Thomsen and S. Reich: Phys. Rev. Lett. 85, 5214 (2000)

J. Maultzsch, S. Reich and C. Thomsen: Phys. Rev. B 64121407 (2001); 65, $233402(2002)$

65. E. Dobardžić, J. Maultzsch, I. Milošević, C. Thomsen and M. Damnjanović: Phys. Status Solidi b 237, R7 (2003)

66. E. Dobardžić, I. Milošević, T. Vuković, B. Nikolić and M. Damnjanović: Eur. Phys. J. B 34, 409 (2003)

M. Damnjanović, E. Dobardžić, I. Milošević, T. Vuković, B. Nikolić: New Journal of Physics 5, (1) 1-15 (2003)

M. Damnjanović, I. Milošević, E. Dobardžić, T. Vuković, B. Nikolić, Phys. Rev. B 69153401 (2004)

67. A. Jishi and G. Dresselhaus: Phys. Rev. B 26, 4514 (1982)

68. V.N. Popov: Phys. Rev. B 66, 153408 (2002)

69. S. Bandow, M. Takizawa, K. Hirahara, M. Yudasaka and S. Iijima: Chem. Phys. Lett. 337, 48 (2001)

70. J.M. Benoit, J.P. Buisson, O. Chauvet, C. Godon and S. Lefrant: Phys. Rev. B 66, 073417 (2002)

71. S. Bandow, G. Chen, G.U. Sumanasekera, R. Gupta, M. Yudasaka, S. Iijima and P.C. Eklund: Phys. Rev. B 66, 075416 (2002)

72. Y.E. Lozovik, A.V. Minogin, A.M. Popov: Phys. Lett. A 313, 112 (2003)

73. M. Damnjanović, T. Vuković and I. Milošević: Eur. Phys. J. B 25, 131 (2002)

T. Vuković, M. Damnjanović and I. Milošević: Physica E 16, 259 (2003)

74. J.C. Charlier and J.P. Michenaud: Phys. Rev. Lett. 70, 1858 (1993)

75. A.H.R. Palser: Phys. Chem. Chem. Phys. 14459 (1999) 
88 M. Damnjanović et al.

76. J. Cumings and A. Zettl: Science 289, 602 (2000)

77. M. Yu, B. Yakobson and R. Ruoff: J. Phys. Chem. B 104, 8764 (2000) 